M. Zhu

Nagoya Math. J.

Vol. 117 (1990), 63-92

\title{
EQUILIBRIUM FLUCTUATIONS FOR ONE-DIMENSIONAL GINZBURG-LANDAU LATTICE MODEL
}

\author{
MING ZHU
}

\section{Dedicated to Professor Takeyuki Hida on his 60th birthday}

\section{§1. Introduction}

We shall investigate a system of spin configurations $S=\{S(t, x) ; t \geq 0$, $x \in \mathbb{Z}\}$ on a one-dimensional lattice $\mathbb{Z}$ changing randomly in time. The evolution law is described by an infinite-dimensional stochastic differential equation (SDE):

$$
\begin{aligned}
d S(t, x)=\{ & \left.U^{\prime}(S(t, x+1))-2 U^{\prime}(S(t, x))+U^{\prime}(S(t, x-1))\right\} d t \\
& +\sqrt{2}(d \beta(t, x+1)-d \beta(t, x)), \quad x \in Z
\end{aligned}
$$

where $\{\beta(t, x) ; t \geq 0, x \in Z\}$ is a family of independent standard Wiener processes and $U^{\prime}$ is the derivative of a self-potential $U: \boldsymbol{R} \rightarrow \boldsymbol{R}$. Throughout this paper we are assuming that $U$ has two times continuous derivatives and

$$
a-A \leq U^{\prime \prime}(x) \leq a+A
$$

with some constants $a>0$ and $A>0$. The system (1.1) is called onedimensional Ginzburg-Landau lattice model (cf. [1], [2]), which has a unique strong solution in a certain class of configuration spaces (see Section 2, Theorem 2.1).

The purpose of the present paper is to investigate the hydrodynamical behavior, especially the equilibrium fluctuation problem, for (1.1). We introduce the space-time scaling:

$$
x \rightarrow[x / \varepsilon], \quad t \rightarrow t / \varepsilon^{2}, \quad \varepsilon>0
$$

for the equation (1.1). Here $[u]$ denotes the integral part of $u \in \boldsymbol{R}$. After this scaling the process $S_{\varepsilon}(t, x)=S\left(t / \varepsilon^{2},[x / \varepsilon]\right)$ solves the following scaled

Received October 28, 1988. 
equation:

$$
d S_{\varepsilon}(t, x)=\Delta_{\varepsilon} U^{\prime}\left(S_{\varepsilon}(t, x)\right) d t+\sqrt{2 \varepsilon} \nabla_{\varepsilon} d w_{\varepsilon}(t, x), \quad t>0, \quad x \in R
$$

where $w_{\varepsilon}(t, x)=\sqrt{\varepsilon} \beta\left(t / \varepsilon^{2},[x / \varepsilon]\right)$ and

$$
\begin{aligned}
& \Delta_{\varepsilon} \varphi(x)=\varepsilon^{-1}[\varphi(x+\varepsilon)-\varphi(x)] \\
& \Delta_{\varepsilon} \varphi(x)=\varepsilon^{-2}[\varphi(x+\varepsilon)-2 \varphi(x)+\varphi(x-\varepsilon)]
\end{aligned}
$$

for functions $\varphi$ of $x$. The operations $\nabla_{\varepsilon}$ and $\Delta_{\varepsilon}$ are the lattice approximations of step size $\varepsilon$ to the differential operators $\partial / \partial x$ and $\partial^{2} / \partial x^{2}$, respectively. We are interested in the asymptotic behavior of $S_{\varepsilon}(t, x)$ as $\varepsilon$ tends to 0 .

Two kinds of problems are formulated concerning the hydrodynamical limit: the law of large numbers and the central limit theorem. For the lattice model (1.1), Fritz [2] proved the law of large numbers in the nonstationary case (in fact, he investigated more general lattice system) and Guo, Papanicolaou and Varadhan [3] gave a quite different approach to the same problem but in a finite volume case. It is known that $S_{\varepsilon}(t, x)$ converges as $\varepsilon \rightarrow 0$ to a deterministic limit $\gamma(t, x)$ which satisfies a diffusion equation

$$
\frac{\partial \gamma}{\partial t}=\frac{\partial}{\partial x}\left[D(\gamma) \frac{\partial \gamma}{\partial x}\right]
$$

with a certain diffusion coefficient $D(\gamma)$.

On the other hand, the equilibrium fluctuation problem which is the main problem of this paper is to investigate the asymptotic behavior of $V_{\varepsilon}(t, x)=\left(S_{\varepsilon}(t, x)-\gamma\right) / \sqrt{\varepsilon}$ for lattice model (1.1) in the stationary case, where $\gamma=E\left[S_{\varepsilon}(t, x)\right]$ is independent of $(t, x)$. The result will be formulated as the central limit theorem for the SDE (1.1). We shall prove that $V_{\varepsilon}=\left(V_{\varepsilon}(t, x) ; t \geq 0, x \in R\right)$ converges as $\varepsilon \rightarrow 0$ to a generalized OrnsteinUhlenbeck process $V(t)$ characterized by an SDE

$$
d V(t)=D(\gamma) \Delta V(t) d t+\sqrt{2} D d w(t)
$$

where the constant $D(\gamma)$ is the same one as in (1.6) (see Section 2, Theorem 2.2 and Remark in detail), $\Delta=\partial^{2} / \partial x^{2}, \nabla=\partial / \partial x$, and $w(t)$ is a cylindrical Brownian motion on $\boldsymbol{L}^{2}(\boldsymbol{R})$. Spohn [10] investigated the equilibrium fluctuation problem for an interacting Brownian particles' model. In this paper we shall follow the method due to Rost [7] and Spohn [10]. 


\section{§2. Main result}

Let $\boldsymbol{R}^{\boldsymbol{Z}}=\left\{\sigma=\left(\cdots, \sigma_{-1}, \sigma_{0}, \sigma_{1}, \cdots\right) ; \sigma_{k} \in \boldsymbol{R}, k \in \boldsymbol{Z}\right\}$ the space with usual product topology and denote its Borel field by $\mathscr{B}\left(\boldsymbol{R}^{\boldsymbol{Z}}\right)$.

Define product measures $\mu_{\lambda}, \lambda \in R$, on $\left(\boldsymbol{R}^{Z}, \mathscr{B}\left(\boldsymbol{R}^{Z}\right)\right)$ by

$$
\mu_{\lambda}(d \sigma)=\prod_{k=-\infty}^{\infty} q_{\lambda}\left(\sigma_{k}\right) d \sigma_{k}
$$

where

$$
q_{\lambda}(x)=M(\lambda)^{-1} \exp [\lambda x-U(x)]
$$

and

$$
M(\lambda)=\int_{R} \exp [\lambda x-U(x)] d x
$$

The probability measure $\mu_{z}$ can be regarded as a Gibbs state associated with the (formal) Hamiltonian:

$$
H_{\lambda}(\sigma)=\sum_{k \in \boldsymbol{Z}} U\left(\sigma_{k}\right)-\lambda \sum_{k \in \boldsymbol{Z}} \sigma_{k} .
$$

We develop some more notation

$$
\begin{gathered}
\rho(\lambda)=\log M(\lambda), \\
h(\gamma)=\sup _{\lambda}[(\lambda \gamma-\rho(\lambda)], \quad \gamma \in \boldsymbol{R} .
\end{gathered}
$$

Then $h($.$) and \rho($.$) are a pair of conjugate convex functions and$

$$
\lambda=h^{\prime}(\gamma) \text { if and only if } \gamma=\rho^{\prime}(\lambda) \text {. }
$$

Elementary calculation shows

$$
\int x q_{\lambda}(x) d x=\rho^{\prime}(\lambda)
$$

Moreover, $\rho^{\prime \prime}(\lambda)$ is the variance of $q_{\lambda}(x) d x$ i.e.

$$
\int\left(x-\rho^{\prime}(\lambda)\right)^{2} q_{\lambda}(x) d x=\rho^{\prime \prime}(\lambda) .
$$

One knows also that $\rho^{\prime}$ and $h^{\prime}$ are smooth strictly increasing functions.

Let $r>0$ be fixed throughout this paper. Let $\xi(x) \in C^{\infty}(\boldsymbol{R})$ be a positive function such that $\xi(x)=|x|$ if $|x| \geq 1$. We define a Hilbert space as 


$$
\boldsymbol{L}_{r}^{2}=\left\{\boldsymbol{\sigma} \in \boldsymbol{R}^{\boldsymbol{Z}} ;|\boldsymbol{\sigma}|_{r}^{2}=\sum_{k \in \boldsymbol{Z}}\left|\sigma_{k}\right|^{2} \int_{k}^{k+1} \exp [-r \xi(x)] d x<\infty\right\}
$$

One can check that $\mu_{\lambda}\left(\boldsymbol{L}_{r}^{2}\right)=1$.

Now we turn to the study of the SDE (1.1). In view of (1.2), the drift term of (1.1) is linearly bounded and uniformly Lipschitz continuous in the space $\boldsymbol{L}_{r}^{2}$. Therefore, a standard argument yields the existence and uniqueness of strong solutions to (1.1) in $\boldsymbol{L}_{r}^{2}$ (cf. [9]):

THEOREM 2.1. For each $\sigma \in L_{r}^{2}$, the SDE (1.1) has a unique $L_{r}^{2}$-valued continuous strong solution $S_{t}$ starting from $\sigma$ (i.e. $S_{0}=\sigma$ ).

Let $T_{t}, t \geq 0$ be defined by

$$
\left(T_{t} F\right)(\sigma)=E_{\sigma}\left[F\left(S_{t}\right)\right], \quad F \in C\left(L_{r}^{2}\right)
$$

where $\boldsymbol{E}_{\sigma}[$ ] means the expectation under the probability law of (1.1)'s solution $S_{t}$ starting from $\sigma \in \boldsymbol{L}_{r}^{2}$. Then we can easily extend $\left\{\boldsymbol{T}_{t}\right\}_{t \geq 0}$ to a self-adjoint strongly continuous contraction semigroup on $L^{2}\left(\boldsymbol{R}^{Z}, \mu_{\lambda}\right)$ and check that the Gibbs states $\mu_{\lambda}, \lambda \in R$, are reversible measures of $T_{t}$.

Let $\mathscr{E}_{r}=\mathscr{S} \exp [-r \xi(x)]$ the nuclear space with a topology introduced from $\mathscr{S}$, where $\mathscr{S}=\mathscr{S}(\boldsymbol{R})$ is Schwartz space. Let $\mathscr{E}_{r}^{\prime}$ be the dual space of $\mathscr{E}_{r}$ with the strong topology and $\mathscr{C}=C\left([0, \infty) ; \mathscr{E}_{r}^{\prime}\right)$. Let $\{S(t, x) ; t \geq 0$, $x \in Z$ \} be the solution of (1.1) with initial distribution $\mu_{2}$. Then by Theorem 2.1, we know $S_{\varepsilon}(t, x)=S\left(t / \varepsilon^{2},[x / \varepsilon]\right)$ is in $\mathscr{C}$ (a.s.). Now we can state our main result:

Theorem 2.2. Let $V_{\varepsilon}(t, x)=\varepsilon^{-1 / 2}\left(S_{\varepsilon}(t, x)-\rho^{\prime}(\lambda)\right)$ and $P_{\varepsilon}$ be the probability distribution of $V_{\varepsilon}$ on $\mathscr{C}$. Then $P_{\varepsilon}$ converges as $\varepsilon \rightarrow 0$ to a distribution of a generalized Ornstein-Uhlenbeck process $V=\left\{V_{t}\right\}_{t>0}$ weakly on $\mathscr{C}$. The process $\left\{V_{t}\right\}$ satisfies the following equation

$$
d V_{t}=\rho^{\prime \prime}(\lambda)^{-1} \Delta V_{t} d t+\sqrt{2} \nabla d w_{t}
$$

where $\Delta=\partial^{2} / \partial x^{2}, \quad \nabla=\partial / \partial x$ and $w_{t}$ is a cylindrical Brownian motion on $\boldsymbol{L}^{2}(\boldsymbol{R})$.

Remark. From the relationship (2.7), we have $\rho^{\prime \prime}(\lambda)^{-1}=h^{\prime \prime}\left(\rho^{\prime}(\lambda)\right)$. However, it is known that $h^{\prime \prime}(\gamma)=D(\gamma)$; the diffusion coefficient appearing in (1.6) (cf. [1] [2] [3]). 


\section{§3. Sketch of the proof and Boltzmann-Gibbs principle}

Let $V_{\varepsilon}$ be the stationary process defined as in Section 2. From (1.4), we get an equation for $V_{\varepsilon}(t, x)$

$$
\begin{gathered}
d V_{\varepsilon}(t, x)=\frac{1}{\sqrt{\varepsilon}} \Delta_{\varepsilon} U^{\prime}\left(\sqrt{\varepsilon} V_{\varepsilon}(t, x)+\rho^{\prime}(\lambda)\right) d t+\sqrt{2} V_{\varepsilon} d w_{\varepsilon}(t, x), \\
x \in R, \quad t>0 .
\end{gathered}
$$

Tending $\varepsilon$ to 0 in (3.1), the second term converges to $\sqrt{2} \nabla d w(t)$ (at least formally). The difficulty in the proof of Theorem 2.2 lies in the computation of the first term. Although it is nonlinear, Rost [7] and [8] suggest that it should converge to a linear term $\rho^{\prime \prime}(\lambda)^{-1} \Delta V(t)$; precisely saying, our goal will be the following:

Proposition 3.1 (Boltzmann-Gibbs principle). For each $t>0$ and $f \in \mathscr{E}_{r}$,

$$
\begin{gathered}
E\left[\left(\int_{0}^{t} d s \int_{R} d x \frac{1}{\sqrt{\varepsilon}}\left\{U^{\prime}\left(S_{\varepsilon}(s, x)\right)-\rho^{\prime \prime}(\lambda)^{-1} S_{\varepsilon}(s, x)\right\} \Delta_{\varepsilon} f(x)\right)^{2}\right] \rightarrow 0, \\
\text { as } \varepsilon \rightarrow 0 .
\end{gathered}
$$

In the rest of this section, we give an outline of the proof of this proposition. For convenience, we intrdoce some notation:

$$
\begin{aligned}
& \Phi(x)=U^{\prime}(x)-\rho^{\prime \prime}(\lambda)^{-1} x, \quad x \in \boldsymbol{R}, \\
& f_{\Delta}^{(\varepsilon)}(x)=\varepsilon^{1 / 2}\left(\Delta_{\varepsilon} f\right)(\varepsilon x), \quad \text { for } f \in \mathscr{E}_{r}, \\
& \Phi(f)(\sigma)=\int_{R} \Phi\left(\sigma_{[x]}\right) f(x) d x, \quad \text { for } f \in \mathscr{E}_{r}, \quad \sigma \in L_{r}^{2}, \\
& \Phi(f, t)=\Phi(f)\left(S_{t}\right), \quad S_{t}=\{S(t, x) ; x \in Z\} \in L_{r}^{2} \text { (a.s.), } t \geq 0, \\
& R(\varepsilon)=E\left[\left(\int_{0}^{t} d s \Phi\left(f_{\Delta}^{(\varepsilon)}, s / \varepsilon^{2}\right)\right)^{2}\right] .
\end{aligned}
$$

It is easy to check that $R(\varepsilon)=$ the 1.h.s. of (3.2). Hence our goal is to show that $\lim _{\varepsilon \rightarrow 0} R(\varepsilon)=0$. We define a class of shift operators $\left\{\tau_{q}\right\}_{q \in \boldsymbol{R}}$ as follows: For $q \in \boldsymbol{R}, \sigma \in \boldsymbol{L}_{r}^{2}$, and any functional $F$ of $\sigma$,

$$
\begin{aligned}
& \left(\tau_{q} \sigma\right)_{x}=\sigma_{[x+q]}, \\
& \left(\tau_{q} F\right)(\sigma)=F\left(\tau_{q} \sigma\right) .
\end{aligned}
$$

Now take $g \in C_{0}^{\infty}(R)$ satisfying $\int g(x) d x=1$ and fix $t>0, f \in \mathscr{E}_{r}$. For every $T, \varepsilon>0$, choose $N=\left[T^{-1} \varepsilon^{-2} t\right]$, then we have from the stationarity of $S(t, x)$ : 


$$
\begin{aligned}
R(\varepsilon) & =\boldsymbol{E}\left[\left(\varepsilon^{2} \int_{0}^{\epsilon-2 t} d s \Phi\left(f_{\Delta}^{(s)}, s\right)\right)^{2}\right] \\
& \leq 2 \varepsilon^{4} E\left[\left(\sum_{n=0}^{N-1} \int_{n T}^{(n+1) T} d s \Phi\left(f_{\Delta}^{(\varepsilon)}, s\right)\right)^{2}\right]+2 \varepsilon^{4} \boldsymbol{E}\left[\left(\int_{N T}^{\epsilon-2 t} d s \Phi\left(f_{\Delta}^{(s)}, s\right)\right)^{2}\right] \\
& \leq 2 \varepsilon^{4} E\left[N \cdot \sum_{n=0}^{N-1}\left(\int_{n T}^{(n+1) T} d s \Phi\left(f_{\Delta}^{(s)}, s\right)\right)^{2}\right]+R_{3}(\varepsilon) \\
& =2 \varepsilon^{4} N^{2} E\left[\left(\int_{0}^{T} d s \Phi\left(f_{\Delta}^{(s)}, s\right)\right)^{2}\right]+R_{3}(\varepsilon) \\
& \leq 2 t^{2} T^{-2} E\left[\int_{0}^{T} d s \int_{0}^{T} d u \Phi\left(f_{\Delta}^{(s)}, s\right) \Phi\left(f_{\Delta}^{(s)}, s\right)\right]+R_{3}(\varepsilon) \\
& \leq R_{1}(\varepsilon)+R_{2}(\varepsilon)+R_{3}(\varepsilon)
\end{aligned}
$$

where

$$
\begin{aligned}
& R_{1}(\varepsilon)=4 t^{2} T^{-2} \int_{0}^{T} d s \int_{0}^{T} d u\left\langle\left(T_{|s-u| / 2} \Phi\left(g * f_{\Delta}^{(s)}\right)\right)^{2}\right\rangle \\
& R_{2}(\varepsilon)=4 t^{2} T^{-2} \int_{0}^{T} d s \int_{0}^{T} d u\left\langle\left(T_{|s-u| / 2} \Phi\left(f_{\Delta}^{(s)}\right)-T_{|s-u| / 2} \Phi\left(g * f_{\Delta}^{(s)}\right)\right)^{2}\right\rangle \\
& R_{3}(\varepsilon)=2 \varepsilon^{4} E\left[\left(\int_{0}^{-2 t-N T} d s \Phi\left(f_{\Delta}^{(\varepsilon)}, s\right)\right)^{2}\right]
\end{aligned}
$$

and $\langle\cdot\rangle$ stands for the expectation with respect to $\mu_{\lambda}$; it will be sometimes denoted by $\langle\cdot\rangle_{\lambda}$ to be made its dependence on $\lambda$ clear (Section 6 ). These three terms can be estimated as follows.

LEMMA 3.2.

(1) If $\int_{R} d q\left|\left\langle\Phi(g) \tau_{q} T_{2 t} \Phi(g)\right\rangle-\langle\Phi(g)\rangle^{2}\right|\langle\infty$, then

$$
\lim _{\varepsilon \rightarrow 0}\left\langle\left(T_{t} \Phi\left(g * f_{\Delta}^{(s)}\right)\right)^{2}\right\rangle=\|\Delta f\|^{2} \int_{R} d q\left\{\left\langle\Phi(g) \tau_{q} T_{2 t} \Phi(g)\right\rangle-\langle\Phi(g)\rangle^{2}\right\} .
$$

(2) $\lim _{s \rightarrow 0}\left\langle\left(T_{t} \Phi\left(g * f_{\Delta}^{(\epsilon)}\right)-T_{t} \Phi\left(f_{\Delta}^{(s)}\right)\right)^{2}\right\rangle=0$, for all $t>0$.

(3) $\lim _{\varepsilon \rightarrow 0} R_{3}(\varepsilon)=0$.

Proof. (1) By the uniqueness of solutions of eq. (1.1), it is easy to see that $T_{t}\left(\tau_{q} \Phi(g)\right)=\tau_{-q}\left(T_{t} \Phi(g)\right)$. Thus

$$
T_{t} \Phi\left(g * f_{\Delta}^{(\varepsilon)}\right)(\sigma)=\int_{R} d q f_{\Delta}^{(\varepsilon)}(q) \tau_{-q} T_{t} \Phi(g) .
$$

Noting that $\left\langle T_{t} \Phi\left(g * f_{\Delta}^{(\delta)}\right)\right\rangle=0$, we have

$$
\begin{aligned}
& \left\langle\left(T_{t} \Phi\left(g * f_{\Delta}^{(\varepsilon)}\right)\right)^{2}\right\rangle=\left\langle\left(T_{t} \Phi\left(g * f_{\Delta}^{(s)}\right)\right)^{2}\right\rangle-\left\langle T_{t} \Phi\left(g * f_{\Delta}^{(\varepsilon)}\right)\right\rangle^{2} \\
& \quad=\int_{R} d p\left(\Delta_{\varepsilon} f\right)(p) \int_{R} d q\left(\Delta_{\varepsilon} f\right)(\varepsilon q+p)\left\{\left\langle\Phi(g) \tau_{q} T_{2 t} \Phi(g)\right\rangle-\langle\Phi(g)\rangle^{2}\right\} .
\end{aligned}
$$


Therefore (3.3) is established by letting $\varepsilon \rightarrow 0$.

(2) We compute

$$
\begin{aligned}
& \left\langle\left(T_{t} \Phi\left(g * f_{\Delta}^{(\varepsilon)}\right)-T_{t} \Phi\left(f_{\Delta}^{(\varepsilon)}\right)\right)^{2}\right\rangle \leq\left\langle\left(\Phi\left(g * f_{\Delta}^{(\varepsilon)}\right)-\Phi\left(f_{\Delta}^{(s)}\right)\right)^{2}\right\rangle \\
& \quad=\left\langle\Phi\left(\sigma_{0}\right)^{2}\right\rangle \int d x d y \mathbf{1}_{\{[x]=[y]\}}\left(g * f_{\Delta}^{(\varepsilon)}-f_{\Delta}^{(\delta)}\right)(x)\left(g * f_{\Delta}^{(\varepsilon)}-f_{\Delta}^{(\varepsilon)}\right)(y) .
\end{aligned}
$$

Besauce the r.h.s. tends to 0 as $\varepsilon \rightarrow 0$, the assertion is proved.

$$
\text { (3) } \begin{aligned}
R_{3}(\varepsilon) & \leq 2 \varepsilon^{4} T E\left[\int_{0}^{s-2 t-N T} d s \Phi^{2}\left(f_{\Delta}^{(\varepsilon)}, s\right)\right]=2 \varepsilon^{4} T \int_{0}^{--2 t-N T} d s\left\langle\Phi^{2}\left(f_{\Delta}^{(s)}\right)\right\rangle \\
& \leq 2 \varepsilon^{4} T^{2}\left\langle\Phi^{2}\left(\sigma_{0}\right)\right\rangle \int d x d y \mathbf{1}_{\{[x]=[y]\}} f_{\Delta}^{(s)}(x) f_{\Delta}^{(\varepsilon)}(y) .
\end{aligned}
$$

Taking the limit $\varepsilon \rightarrow 0$ proves the conclusion.

This lemma shows

$$
\lim _{c \rightarrow 0} R(\varepsilon) \leq 4 t^{2} T^{-2}\|\Delta f\|^{2} \cdot \int_{0}^{T} d s \int_{0}^{T} d u \int d q\left\{\left\langle\Phi(g) \tau_{q} T_{|s-u|} \Phi(g)\right\rangle-\langle\Phi(g)\rangle^{2}\right\} .
$$

Hence, it is sufficient to show that

$$
\lim _{T \rightarrow \infty} T^{-2} \cdot \int_{0}^{T} d s \int_{0}^{T} d u \int d q\left\{\left\langle\Phi(g) \tau_{q} T_{|s-u|} \Phi(g)\right\rangle-\langle\Phi(g)\rangle^{2}\right\}=0 .
$$

Clearly, this is equivalent to the following statement:

$$
\lim _{t \rightarrow \infty} \int_{R} d q\left\{\left\langle\Phi(g) \tau_{q} T_{t} \Phi(g)\right\rangle-\langle\Phi(g)\rangle^{2}\right\}=0
$$

However a simple calculation proves

$$
\int d q\left\{\left\langle\Phi(g) \tau_{q} T_{t} \Phi(g)\right\rangle-\langle\Phi(g)\rangle^{2}\right\}=\sum_{n=-\infty}^{\infty}\left\{\left\langle\Phi(g) \tau_{n} T_{t} \Phi(g)\right\rangle-\langle\Phi(g)\rangle^{2}\right\}, \quad t \geq 0 .
$$

Therefore (3.5) is equivalent to its lattice form:

$$
\lim _{t \rightarrow \infty} \sum_{n=-\infty}^{\infty}\left\{\left\langle\Phi(g) \tau_{n} T_{t} \Phi(g)\right\rangle-\langle\Phi(g)\rangle^{2}\right\}=0 .
$$

Now, we introduce a Hilbert space $\mathscr{H}$ with inner product $\langle F \mid G\rangle=$ $\sum_{n=-\infty}^{\infty}\left\{\left\langle F \tau_{n} G\right\rangle-\langle F\rangle\langle G\rangle\right\}, F, G \in \mathscr{H}$. This space will be discussed in detail in Section 4. By Proposition 6.2, $T_{t}$ is ergodic in $\mathscr{H}$, and

$$
\lim _{t \rightarrow \infty} T_{t} F=\rho^{\prime \prime}(\lambda)^{-1}\left\langle F \mid F_{0}(g)\right\rangle F_{0}(g) \quad \text { in } \mathscr{H}, \text { for } F \in \mathscr{H}
$$

where $F_{0}(g)=\int\left(\sigma(x)-\rho^{\prime}(\lambda)\right) g(x) d x$. Therefore 


$$
\lim _{t \rightarrow \infty} \sum_{n=-\infty}^{\infty}\left\{\left\langle\Phi(g) \tau_{n} T_{t} \Phi(g)\right\rangle-\langle\Phi(g)\rangle^{2}\right\}=\rho^{\prime \prime}(\lambda)^{-1}\left\langle\Phi(g) \mid F_{0}(g)\right\rangle^{2} .
$$

A simple calculation shows that $\left\langle\Phi(g) \mid F_{0}(g)\right\rangle=0$. Consequently, we establish (3.6). Thus Boltzmann-Gibbs principle is shown.

The definition of the Hilbert space $\mathscr{H}$ and the ergodicity of $T_{t}$ in $\mathscr{H}$ will be dealt with in Sections 4,5 and 6 . The martingale approach will be applied for showing the main theorem in Sections 7 and 8 .

\section{$\S 4$. Construction of the Hilbert space $\mathscr{H}$}

As explained in Section 3, we want to introduce a Hilbert space $\mathscr{H}$ with the inner product $\langle\cdot \mid \cdot\rangle$. In this section, we shall define the space $\mathscr{H}$ by completing a class of local functions and investigate the relation between the $L^{2}$-norm approximation and the $\mathscr{H}$-norm approximation.

First we define the classes of local functions:

$$
\begin{aligned}
& \mathscr{F}_{2,[-k, k]}=\left\{F\left(\sigma_{-k}, \cdots, \sigma_{k}\right): F \in L^{2}\left(R^{2 k+1}, \prod_{i=-k}^{k} q_{\lambda}\left(\sigma_{i}\right) d \sigma_{i}\right)\right\} \\
& \mathscr{F}_{2,1 \mathrm{loc}}=\bigcup_{k \in Z^{+}} \mathscr{F}_{2,[-k, k]}
\end{aligned}
$$

LEMMA 4.1. Assume $F_{i} \in \mathscr{F}_{2,100}$ satisfy $\left\langle F_{i}\right\rangle=0, i=1,2$. Then,

$$
\begin{aligned}
& \text { (1) } \sum_{n=-\infty}^{\infty}\left|\left\langle F_{1} \tau_{n} F_{1}\right\rangle\right| \leq(4 \alpha+1)\left\langle F_{1}^{2}\right\rangle<\infty, \text { if } F_{1} \in \mathscr{F}_{2,[-\alpha, \alpha]}, \quad \alpha \in Z^{+} \\
& \text {(2) } \sum_{n=-\infty}^{\infty}\left\langle F_{1} \tau_{n} F_{2}\right\rangle=\lim _{n \rightarrow \infty} \frac{1}{2 n+1}\left\langle\left(\sum_{k=-n}^{n} \tau_{k} F_{1}\right)\left(\sum_{k=-n}^{n} \tau_{k} F_{2}\right)\right\rangle \\
& \text { (3) } \sum_{n=-\infty}^{\infty}\left\langle F_{1} \tau_{n} F_{1}\right\rangle \geq 0
\end{aligned}
$$

Proof. (1) Since $F_{1}(\sigma)=F_{1}\left(\sigma_{-\alpha}, \cdots, \sigma_{\alpha}\right) \in \mathscr{F}_{2,[-\alpha, \alpha]}$, we have

$$
\begin{aligned}
\sum_{n=-\infty}^{\infty}\left|\left\langle F_{1} \tau_{n} F_{1}\right\rangle\right| & =\sum_{n=-2 \alpha}^{2 \alpha}\left|\left\langle F_{1}\left(\sigma_{-\alpha}, \cdots, \sigma_{\alpha}\right) F_{1}\left(\sigma_{-\alpha+n}, \cdots, \sigma_{\alpha+n}\right)\right\rangle\right| \\
& \leq \sum_{n=-2 \alpha}^{2 \alpha}\left\langle F_{1}\left(\sigma_{-\alpha}, \cdots, \sigma_{\alpha}\right)^{2}\right\rangle^{1 / 2}\left\langle F_{1}\left(\sigma_{-\alpha+n}, \cdots, \sigma_{\alpha+n}\right)^{2}\right\rangle^{1 / 2} \\
& =(4 \alpha+1)\left\langle F_{1}^{2}\right\rangle
\end{aligned}
$$

(2) First we note

$$
\sum_{n=-2 n}^{2 n}\left\langle F_{1} \tau_{k} F_{2}\right\rangle=\frac{1}{2 n+1}\left\langle\left(\sum_{k=-n}^{n} \tau_{k} F_{1}\right)\left(\sum_{l=-n}^{n} \tau_{l} F_{2}\right)\right\rangle+R(n),
$$

where 


$$
R(n)=(2 n+1)^{-1} \sum_{k=1}^{2 n} k\left(\left\langle\tau_{k} F_{1} F_{2}\right\rangle+\left\langle F_{1} \tau_{k} F_{2}\right\rangle\right)
$$

However, since $F_{1}, F_{2} \in \mathscr{F}_{2,[-\beta, \beta]}$ with some $\beta \in Z^{+},\left\langle\tau_{k} F_{1} F_{2}\right\rangle=\left\langle F_{1} \tau_{k} F_{2}\right\rangle=$ $\left\langle F_{1}\right\rangle\left\langle F_{2}\right\rangle=0$ for $k>2 \beta$. Therefore

$$
|R(n)| \leq \frac{1}{2 n+1} \sum_{k=1}^{2 \beta} k\left|\left\langle\tau_{k} F_{1} F_{2}\right\rangle+\left\langle F_{1} \tau_{k} F_{2}\right\rangle\right| \rightarrow 0, \quad \text { as } n \rightarrow \infty .
$$

Taking the limit $n \rightarrow \infty$ in (4.4), we prove (4.2).

(3) is consequence of (4.2).

Lemma 4.1 enables us to define the Hilbert space $\mathscr{H}$ :

Definition. For $F_{1}, F_{2} \in \mathscr{F}_{2,10 c}$, set

$$
\left\langle F_{1} \mid F_{2}\right\rangle=\sum_{n=-\infty}^{\infty}\left(\left\langle F_{1} \tau_{n} F_{2}\right\rangle-\left\langle F_{1}\right\rangle\left\langle F_{2}\right\rangle\right) .
$$

We define the Hilbert space $\mathscr{H}$ as the completion of $\mathscr{F}_{2,10 \mathrm{c}}$ with inner product $\langle\cdot \mid \cdot\rangle$ modulo $\{F:\langle F \mid F\rangle=0\}$. We shall denote the norm corresponding to $\langle\cdot \mid \cdot\rangle$ by $\|\cdot\|_{\circledast \bullet}$.

Finally, we discuss the relationship between the convergences in two spaces $\boldsymbol{L}^{2}\left(\boldsymbol{R}^{Z}, \mu_{\lambda}\right)$ and $\mathscr{H}$.

LemMA 4.2. Suppose $F_{n} \in \mathscr{F}_{2,[-n, n]}$ satisfies

$$
\lim _{n \rightarrow \infty} n\left\langle F_{n}^{2}\right\rangle=0 \text {. }
$$

Then

$$
\lim _{n \rightarrow \infty}\left\langle F_{n} \mid F_{n}\right\rangle=0 .
$$

Proof. The conclusion follows since Lemma 4.1 (1) implies

$$
\begin{aligned}
0 \leq\left\langle F_{n} \mid F_{n}\right\rangle & =\sum_{k=-\infty}^{\infty}\left\langle\left(F_{n}-\left\langle F_{n}\right\rangle\right) \tau_{k}\left(F_{n}-\left\langle F_{n}\right\rangle\right)\right\rangle \\
& \leq(4 n+1)\left\langle\left(F_{n}-\left\langle F_{n}\right\rangle\right)^{2}\right\rangle \leq(4 n+1)\left\langle F_{n}^{2}\right\rangle .
\end{aligned}
$$

Lemma 4.3. Suppose $F_{1}, F_{2} \in \mathscr{H}$ satisfy $\left\langle F_{1}\right\rangle=\left\langle F_{2}\right\rangle=0$ and $\sum_{n=-\infty}^{\infty} n\left|\left\langle F_{1} \tau_{n} F_{2}\right\rangle\right|<\infty$. Then

$$
\left\langle F_{1} \mid F_{2}\right\rangle=\lim _{n \rightarrow \infty} \frac{1}{2 n+1}\left\langle\left(\sum_{k=-n}^{n} \tau_{k} F_{1}\right)\left(\sum_{k=-n}^{n} \tau_{k} F_{2}\right)\right\rangle .
$$

Proof. This is a consequence of (4.4) and (4.5). 
LEMMA 4.4. Let $F \in L^{2}\left(R^{Z}, \mu_{\lambda}\right)$ and assume there exists $F_{n} \in \mathscr{F}_{2,[-n, n]}$ satisfying $\left\langle F_{n}\right\rangle=0, n=1,2, \cdots$, and $\delta>2$ such that

$$
\left\langle\left(F_{n}-F\right)^{2}\right\rangle \leq C n^{-\delta}
$$

with $C$ independent of $n$. Then

$$
\left|\left\langle F \tau_{k} F\right\rangle\right| \leq C^{\prime}(1+|k|)^{1-\delta / 2}, \quad k \in Z
$$

where $C^{\prime}$ is independent of $k$. Moreover if $\delta>4, F=\lim _{n \rightarrow \infty} F_{n}$ in $\mathscr{H}$ and therefore $F \in \mathscr{H}$.

Proof. Let $G_{1}=F_{1}$ and $G_{n}=F_{n}-F_{n-1}, n=2,3, \cdots$ Then by (4.10) $F=\sum_{n=1}^{\infty} G_{n}$ in $L^{2}\left(\boldsymbol{R}^{Z}, \mu_{\lambda}\right)$ and there exists a constant $C_{1}>0$ such that

$$
\left\langle G_{n}^{2}\right\rangle^{1 / 2} \leq C_{1} n^{-\delta / 2}
$$

Note that $m+n\left\langle|k|\right.$ implies $\left\langle G_{n} \tau_{k} G_{m}\right\rangle=\left\langle G_{n}\right\rangle\left\langle\tau_{k} G_{m}\right\rangle=0$. We can therefore compute by Schwarz inequality and (4.12)

$$
\begin{aligned}
\left|\left\langle F \tau_{k} F\right\rangle\right| & \leq \sum_{n=1}^{\infty} \sum_{m=1}^{\infty}\left|\left\langle G_{n} \tau_{k} G_{m}\right\rangle\right| \leq C_{1}^{2} \sum_{n+m \geq|k|}^{\infty} m^{-\delta / 2} n^{-\delta / 2} \\
& \leq C^{\prime}(1+|k|)^{1-\delta / 2}
\end{aligned}
$$

where $C^{\prime}$ is independent of $k$. Thus (4.11) is established and we also have

$$
\sum_{n=1}^{\infty} \sum_{m=1}^{\infty}\left|\left\langle G_{n} \tau_{k} G_{m}\right\rangle\right| \leq C^{\prime}(1+|k|)^{1-\delta / 2} .
$$

Finally, by (4.12) and (4.13) we have

$$
\begin{aligned}
\| F_{N}-\left.F\right|_{\mathscr{x}} ^{2} & \leq \sum_{|k| \leq N}^{\infty} \sum_{n=N+1}^{\infty} \sum_{m=N+1}^{\infty}\left|\left\langle G_{n} \tau_{k} G_{m}\right\rangle\right|+\sum_{|k| \geq N+1} \sum_{n=1}^{\infty} \sum_{m=1}^{\infty}\left|\left\langle G_{n} \tau_{k} G_{m}\right\rangle\right| \\
& \leq C_{1}^{2} 2 N\left(\sum_{n=N+1}^{\infty} n^{-\delta / 2}\right)^{2}+C^{\prime} \sum_{|k| \geq N+1}(1+|k|)^{1-\delta / 2} .
\end{aligned}
$$

If $\delta>4$, then $\lim _{N \rightarrow \infty} N \sum_{n=N+1}^{\infty} n^{-\delta / 2}=0$ and $\sum_{k \in Z}(1+|k|)^{1-\delta / 2}<\infty$. Consequently, the r.h.s. of (4.14) tends to 0 as $N \rightarrow \infty$. Therefore $\lim _{N \rightarrow \infty} F_{N}=F$ in $\mathscr{H}$ and $F \in \mathscr{H}$.

\section{$\S 5$. Semigroup and its generator in $\mathscr{H}$}

In this section we shall discuss properties of the semigroup $T_{t}$ and its generator $L$, which will be defined in $\mathscr{H}$. We define a class of nice functions, which will be the core for $L$ :

$$
\mathscr{D}_{0} \equiv\left\{F(\sigma)=F\left(\sigma_{-m}, \cdots, \sigma_{m}\right) \in \mathscr{F}_{2,[-m, m]}: F \in C_{0}^{\infty}\left(R^{2 m+1}\right), m \in N\right\} .
$$


Lemma 5.1. $\mathscr{D}_{0}$ is dense in $\mathscr{H}$.

Proof. Since $\mathscr{D}_{0}$ is dense in $L^{2}=L^{2}\left(R^{Z}, \mu_{\lambda}\right)$, we have

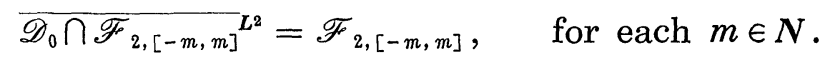

By Lemma 4.1(1)

$$
\langle F \mid F\rangle \leq(4 m+1)\left\langle F^{2}\right\rangle, \quad \text { for } F \in \mathscr{F}_{2,[-m, m]} .
$$

Hence, (5.2) implies that

$$
\overline{\mathscr{D}}_{0} \cap \mathscr{F}_{2,[-m, m]}{ }^{*}=\mathscr{F}_{2,[-m, m]}, \quad \text { for each } m \in N .
$$

Thus

$$
\begin{aligned}
\overline{\mathscr{D}}_{0} & =\overline{\bigcup_{m \in N}\left(\mathscr{D}_{0} \cap \mathscr{F}_{2,[-m, m]}\right)} \supset \bigcup_{m \in N} \overline{\mathscr{D}_{0} \cap \mathscr{F}_{2,[-m, m]}}=\bigcup_{m \in N} \mathscr{F}_{2,[-m, m]} \\
& =\mathscr{F}_{2,10 c} \quad \text { in } \mathscr{H} .
\end{aligned}
$$

Therefore, $\mathscr{H}=\overline{\mathscr{F}_{2,1 \text { loc }}} \subset \overline{\mathscr{D}_{0}}=\overline{\mathscr{D}}_{0}$.

Now we discuss the properties of $T_{t}$ and $L$. First, we show that the function $T_{t} F$ with $F \in \mathscr{D}_{0}$ is in $\mathscr{H}$. To this end, consider the following local SDE's on $[-n, n]$ : For each $n \in N$,

$$
\left\{\begin{aligned}
& d S(t,-n)=\left\{U^{\prime}(S(t,-n+1))\right.\left.-2 U^{\prime}(S(t,-n))+U^{\prime}(S(t, n))\right\} d t \\
&+\sqrt{2}(d \beta(t,-n+1)-d \beta(t,-n)) \\
& d S(t, k)=\Delta_{1} U^{\prime}(S(t, k)) d t+ \sqrt{2} \nabla_{1} d \beta(t, k), k=-n+1, \cdots, n-1 \\
& d S(t, n)=\left\{U^{\prime}(S(t,-n))-2 U^{\prime}(S(t, n))+U^{\prime}(S(t, n-1)\} d t\right. \\
&+\sqrt{2}(d \beta(t,-n)-d \beta(t, n))
\end{aligned}\right.
$$

where $\Delta_{1} a_{k}=a_{k+1}-2 a_{k}+a_{k-1}$ and $\nabla_{1} a_{k}=a_{k+1}-a_{k}$, for sequence $\left\{a_{k}\right\}$. The generator of the process determined by the SDE (5.3) is denoted by $L_{n}$ with domain $\mathscr{D}\left(L_{n}\right)$ and the corresponding semigroup by $T_{t, n}=e^{L_{n} t}$. Then

$$
L_{n}=\sum_{i=-n}^{n}\left(\nabla_{1} \frac{\partial}{\partial \sigma_{i}}\right)^{2}-\sum_{i=-n}^{n} \nabla_{1} U^{\prime}\left(\sigma_{i}\right) \nabla_{1} \frac{\partial}{\partial \sigma_{i}}
$$

where $\partial / \partial \sigma_{n+1} \equiv \partial / \partial \sigma_{-n}$, and $\sigma_{n+1}=\sigma_{-n}$. Note that $\mu_{\lambda}^{(n)}\left(d \sigma_{-n} \cdots d \sigma_{n}\right)=$ $\prod_{k=-n}^{n} q_{\lambda}\left(\sigma_{k}\right) d \sigma_{k}, \lambda \in R$, are the reversible measures of the SDE (5.3).

Lemma 5.2. Let $F \in \mathscr{D}_{0}$ satisfy $\langle F\rangle=0$. Then, for every $t_{0}>0$ and $\delta>0$, there exists a constant $C$ such that

$$
\left|\left\langle F \tau_{k} T_{t} F\right\rangle\right| \leq C(1+|k|)^{-\delta}, \quad \text { for } k \in Z, \quad t \in\left[0, t_{0}\right] .
$$


Moreover, $T_{t, n} F \rightarrow T_{t} F$ is $\mathscr{H}$ as $n \rightarrow \infty$ and especially $T_{t} F \in \mathscr{H}$.

Proof. Let $S(t, \sigma)=\{S(t, k, \sigma)\}_{k \in \boldsymbol{Z}}$ be the solution of (1.1) with initial value $\sigma=\left\{\sigma_{k}\right\}_{k \in Z}$ and $S^{(n)}(t, \sigma)=\left\{S^{(n)}(t, k, \sigma)\right\}_{k=-n}^{n}$ the solution of (5.3) with initial value $\left\{\sigma_{k}\right\}_{k=-n}^{n}$. Since $F \in \mathscr{D}_{0}$ has a form $F(\sigma)=F\left(\sigma_{-\alpha}, \cdots, \sigma_{\alpha}\right)$, with some $\alpha \in N$, we see for $n \geq \alpha$

$$
\begin{aligned}
\left|T_{t, n} F-T_{t} F\right| & =\left|\boldsymbol{E}\left[F\left(S^{(n)}(t, \sigma)\right)\right]-\boldsymbol{E}[F(S(t, \sigma))]\right| \\
& \leq C_{F} \sup _{k \in[-\alpha, \alpha]} E\left[\left|S^{(n)}(t, k, s)-S(t, k, \sigma)\right|\right]
\end{aligned}
$$

where $C_{F}=\sum_{i=-\alpha}^{\alpha}\left\|\frac{\partial F}{\partial \sigma_{i}}\right\|_{\infty}$. Now we set

$$
I_{m}(t)=\sup _{k \in[-\alpha-m, \alpha+m]} E\left[\left|S^{(n)}(t, k, \sigma)-S(t, k, \sigma)\right|^{2}\right] .
$$

Then for every $m: 0 \leq m \leq n-\alpha-2$ and $t_{m}: 0<t_{m} \leq t$,

$$
\begin{gathered}
I_{m}\left(t_{m}\right)=\sup _{k \in[-\alpha-m, \alpha+m]} E\left[\mid \int_{0}^{t_{m}}\left\{\Delta_{1} U^{\prime}\left(S^{(n)}\left(t_{m+1}, k, \sigma\right)\right)\right.\right. \\
\left.\left.-\Delta_{1} U^{\prime}\left(S\left(t_{m+1}, k, \sigma\right)\right)\right\}\left.d t_{m+1}\right|^{2}\right] \\
\leq 16(a+A)^{2} t \int_{0}^{t_{m}} d t_{m+1} I_{m+1}\left(t_{m+1}\right) .
\end{gathered}
$$

Consequenly,

$$
I_{0}(t) \leq\left(16(a+A)^{2} t\right)^{n-\alpha-1} \int_{0}^{t} d t_{1} \int_{0}^{t_{1}} d t_{2} \cdots \int_{0}^{t_{n-\alpha-2}} d t_{n-\alpha-1} I_{n-\alpha-1}\left(t_{n-\alpha-1}\right) .
$$

Noting that

$$
\begin{aligned}
\left\langle I_{n-\alpha-1}(s)\right\rangle & \leq \int d \mu_{2} \sum_{k=-n+1}^{n-1} 2 E\left[S^{(n)}(s, k, \sigma)^{2}+S(s, k, \sigma)^{2}\right] \\
& =4 M_{2}(\lambda)(2 n-1),
\end{aligned}
$$

where $M_{2}(\lambda)=\int_{R} x^{2} q_{\lambda}(x) d x$, we have

$$
\begin{aligned}
\left\langle\left(T_{t, n} F-T_{t} F\right)^{2}\right\rangle & \leq C_{F}^{2}\left\langle I_{0}(t)\right\rangle \\
& \leq 4 C_{F}^{2} M_{2}(\lambda)(2 n-1)\left(16(a+A)^{2} t\right)^{n-\alpha-1} t^{n-\alpha-1} /(n-\alpha-1) !
\end{aligned}
$$

This implies that there exists a constant $C_{1}$ such that

$$
\left\langle\left(T_{t, n} F-T_{t} F\right)^{2}\right\rangle \leq C_{1} n^{-\delta}, \quad \text { for each } \delta \in Z^{+} .
$$

Lemma 4.4 gives an estimate on $\left\langle T_{t} F \tau_{k} T_{t} F\right\rangle$ and therefore on $\left\langle F \tau_{k} T_{t} F\right\rangle$ by replacing $t$ by $t / 2$. This completes the proof. 
Since (5.5) verifies that $\sum_{k=-\infty}^{\infty} k\left|\left\langle T_{t} F \tau_{k} T_{t} F\right\rangle\right|<\infty$ for $F \in \mathscr{D}_{0}$, by Lemma 4.3, we obtain the following form of $\left\langle T_{t} F \mid T_{t} F\right\rangle$ :

$$
\left\langle T_{t} F \mid T_{t} F\right\rangle=\lim _{n \rightarrow \infty} \frac{1}{2 n+1}\left\langle\left(\sum_{k=-n}^{n} \tau_{k} T_{t} F\right)^{2}\right\rangle
$$

Proposition 5.3. $\quad T_{t}$ can be extended uniquely to a strongly continuous self-adjoint contraction semigroup on $\mathscr{H}$.

Proof. By (5.6), for each $t>0$ and $F \in \mathscr{D}_{0}$ satisfying $\langle F\rangle=0$,

$$
\left\|T_{t} F\right\|_{\mathscr{H}}^{2}=\lim _{n \rightarrow \infty} \frac{1}{2 n+1}\left\|T_{t}\left(\sum_{k=-n}^{n} \tau_{-k} F\right)\right\|_{L^{2}}^{2} \leq \lim _{n \rightarrow \infty} \frac{1}{2 n+1}\left\|\sum_{k=-n}^{n} \tau_{-k} F\right\|_{L^{2}}^{2}=\|F\|_{\mathscr{C}}^{2} \text {. }
$$

Thus $\left\|T_{t} F\right\|_{\mathscr{H}} \leq\|F\|_{\mathscr{H}}$ for all $F \in \mathscr{D}_{0}$. We can therefore extend $T_{t}$ from $\mathscr{D}_{0}$ to $\mathscr{H}$ in such a manner that

$$
\left\|T_{t} F\right\|_{\mathscr{H}} \leq\|F\|_{\mathscr{H}} \quad \text { for all } F \in \mathscr{H} \text {. }
$$

It is easy to check that for $F, G \in \mathscr{D}_{0}$,

$$
\left\langle F \mid T_{t} G\right\rangle=\left\langle T_{t} F \mid G\right\rangle \text {. }
$$

This implies the symmetry of $T_{t}$ with the help of (5.7).

Finally we show the strong continuity of $T_{t}$, i.e.

$$
\left\|T_{t} F-F\right\|_{\mathscr{H}} \rightarrow 0, \text { as } t \rightarrow 0, \text { for all } F \in \mathscr{H} \text {. }
$$

In fact, it is enough to show that (5.9) holds for $F \in \mathscr{D}_{2}$; use (5.7) noting that $\mathscr{D}_{0}$ is dense in $\mathscr{H}$. We see from (5.5) that for each $F \in \mathscr{D}_{0}$,

$$
\begin{aligned}
\left|\left\langle\left(T_{t} F-F\right) \tau_{k} F\right\rangle\right| & \leq \mid\left\langle T T_{t} F \tau_{k} F|+|\left\langle F \tau_{k} F\right\rangle\right| \\
& \leq C(1+|k|)^{-2}+\left|\left\langle F \tau_{k} F\right\rangle\right|, \quad k \in Z,
\end{aligned}
$$

and the r.h.s. is summable in $k$. Moreover, we know that $\left\langle\left(T_{t} F-F\right) \tau_{k} F\right\rangle$ $\rightarrow 0$ as $t \rightarrow 0$ by the fact $T_{t}$ is $L^{2}$-strongly continuous. Thus Lebesgue's dominated convergence theorem proves

$$
\left\langle\left(T_{t} F-F\right) \mid F\right\rangle=\sum_{k=-\infty}^{\infty}\left\langle\left(T_{t} F-F\right) \tau_{k} F\right\rangle \rightarrow 0, \text { as } t \rightarrow 0 .
$$

Consequently, we obtain (5.9) for $F \in \mathscr{D}_{0}$ by noting

$$
\left\|T_{t} F-F\right\|_{\mathscr{t}}^{2}=\left\langle T_{2 t} F \mid F\right\rangle-2\left\langle T_{t} F \mid F\right\rangle+\langle F \mid F\rangle \text {. }
$$

Let $L$ be the generator of $T_{t}$ in $\mathscr{H}$. Its domain is denoted by $\mathscr{D}(L)$. We shall see that $L$ has the same form on $\mathscr{D}_{0}$ as the generator of $T_{t}$ in $L^{2}$. 
Lemma 5.4. We have $\mathscr{D}_{0} \subset \mathscr{D}(L)$ and, for every $F(\sigma)=F\left(\sigma_{-\alpha}, \cdots, \sigma_{\alpha}\right) \in \mathscr{D}_{0}$,

$$
(L F)(\sigma)=-\sum_{k \in Z} e^{U^{\prime}\left(\sigma_{k}\right)} \frac{\partial}{\partial \sigma_{k}}\left\{e^{-U^{\prime}\left(\sigma_{k}\right)}\left(\frac{\partial F}{\partial \sigma_{k+1}}-2 \frac{\partial F}{\partial \sigma_{k}}-\frac{\partial F}{\partial \sigma_{k-1}}\right)\right\}
$$

Proof. Let $L^{\prime}$ be the generator of $T_{t}$ in $L^{2}$. We know $\mathscr{D}_{0}$ is in the domain of $L^{\prime}$ and on $\mathscr{D}_{0}, L^{\prime}$ is given by (5.10). Thus $L^{\prime} F \in \mathscr{F}_{2,1 \text { oc }} \subset \mathscr{H}$ for $F \in \mathscr{D}_{0}$, and $\left\|T_{t} L^{\prime} F-L^{\prime} F\right\|_{\mathscr{H}} \rightarrow 0$ as $t \rightarrow 0$. Moreover,

$$
T_{t} F-F=\int_{0}^{t} d s T_{s} L^{\prime} F, \quad \mu_{\lambda}-\text { a.e. }
$$

Therefore

$$
\begin{aligned}
\left\|\frac{1}{t}\left(T_{t} F-F\right)-L^{\prime} F\right\|_{\mathscr{\ell}} & =\left\|\frac{1}{t} \int_{0}^{t} d s\left(T_{s} L^{\prime} F-L^{\prime} F\right)\right\|_{\mathscr{H}} \\
& \leq \frac{1}{t} \int_{0}^{t} d s\left\|T_{s} L^{\prime} F-L^{\prime} F\right\|_{\mathscr{H}} \rightarrow 0 \text { as } t \rightarrow 0 .
\end{aligned}
$$

This means that $L F=L^{\prime} F$.

We shall see that $\mathscr{D}_{0}$ is a domain of essential self-adjointness for $L$ in the following weak sense:

Proposition 5.5. Let $F \in \mathscr{D}(L)$. Then there exist $F_{n} \in \mathscr{D}_{0}$ such that

$$
\lim _{n \rightarrow \infty} F_{n}=F \quad \text { in } \mathscr{H}
$$

and

$$
\lim _{n \rightarrow \infty}\left\langle F_{n} \mid L F_{n}\right\rangle=\langle F \mid L F\rangle .
$$

The first task for the proof of this proposition is to derive the following estimates.

Lemma 5.6. Let $F=F\left(\sigma_{-\alpha}, \cdots, \sigma_{\alpha}\right) \in \mathscr{D}_{0}$. Then for $n \geq \alpha$

$$
\left|\frac{\partial T_{t, n} F}{\partial \sigma_{l}}\right| \leq \begin{cases}C_{F} e^{8(a+A) t} & \text { if }|l| \leq \alpha, \\ C_{F} e^{8(a+A) t}(4(a+A) t)^{|l|-\alpha} /(|l|-\alpha) ! & \text { if } \alpha<|l| \leq n\end{cases}
$$

where $C_{F}=\sum_{k=-\alpha}^{\alpha}\left\|\frac{\partial F}{\partial \sigma_{k}}\right\|_{\infty}$.

Proof. For every $\varepsilon>0$ and $\sigma=\left\{\sigma_{k}\right\} \in \boldsymbol{L}_{r}^{2}$, set $\sigma(l, \varepsilon)=\left\{\sigma_{k}+\delta_{k l} \varepsilon\right\}$ and $\sigma_{[-n, n]}=\left\{\sigma_{-n}, \cdots, \sigma_{n}\right\}$. Then 


$$
\begin{aligned}
& \left|T_{t, n} F\left(\sigma(l, \varepsilon)_{[-n, n]}\right)-T_{t, n} F\left(\sigma_{[-n, n]}\right)\right| \\
& \quad=\left|E\left[F\left(S^{(n)}(t, \sigma(l, \varepsilon))\right)-F\left(S^{(n)}(t, \sigma)\right)\right]\right| \\
& \left.\quad \leq C_{F} \sup _{k \in[-\alpha, \alpha]} E\left[\mid S^{(n)}(t, k, \sigma(l, \varepsilon))-S^{(n)}(t, k, \sigma)\right) \mid\right]
\end{aligned}
$$

where $S^{(n)}$ is defined as in the proof of Lemma 5.2. To get further estimates on the r.h.s. of (5.14), set

$$
\begin{array}{r}
\left.J_{m}^{\varepsilon}(t)=\sup _{k \in[-\alpha-m, \alpha+m]} E\left[\mid S^{(n)}(t, k, \sigma(l, \varepsilon))-S^{(n)}(t, k, \sigma)\right) \mid\right] \\
\text { for } m=0,1, \cdots, n-\alpha .
\end{array}
$$

We have, from the $\operatorname{SDE}$ (5.3), for $m=0,1, \cdots, n-\alpha-1$

$$
\begin{aligned}
J_{m}^{\varepsilon}(t) & =\sup _{k \in[-\alpha-m, \alpha+m]} E\left[\mid \delta_{k \ell} \varepsilon+\int_{0}^{t}\left\{A_{1} U^{\prime}\left(S^{(n)}(s, k, \sigma(l, \varepsilon))\right)\right.\right. \\
& \left.\left.-\Delta_{1} U^{\prime}\left(S^{(n)}(t, k, \sigma)\right)\right\} d s \mid\right] \\
& \leq\left\{\begin{array}{lc}
4(a+A) \int_{0}^{t} d s J_{m+1}^{\varepsilon}(s), & \text { if }|l|>\alpha+m, \\
\varepsilon+4(a+A) \int_{0}^{t} d s J_{m+1}^{\varepsilon}(s), & \text { if }|l| \leq \alpha+m .
\end{array}\right.
\end{aligned}
$$

For $m=n-\alpha$, similarly, we have

$$
J_{n-\alpha}^{\varepsilon}(t) \leq \varepsilon+4(a+A) \int_{0}^{t} d s J_{n-\alpha}^{\varepsilon}(s) .
$$

This implies with the help of Gronwall's lemma

$$
J_{n-\alpha}^{\varepsilon}(t) \leq \varepsilon e^{4(a+A) t} .
$$

Therefore, combining (5.14), (5.16) and (5.18), we can easily show that the 1.h.s. of (5.14) divided by $\varepsilon$ is bounded by the r.h.s. of (5.13) for every $l$; $|l| \leq n$.

Proof of Proposition 5.5. Since the space $\cup_{t \geq 0} T_{t} \mathscr{D}_{0}$ is a core for $L$ (see Reed and Simon [6], II. Th. X. 49), the proof is completed if for every $F=F\left(\sigma_{-\alpha}, \cdots, \sigma_{\alpha}\right) \in \mathscr{D}_{0}$ and $t \geq 0$, we can find functions $F_{n} \in \mathscr{D}_{0}$ such that

$$
\lim _{n \rightarrow \infty} F_{n}=T_{t} F \quad \text { in } \mathscr{H}
$$

and

$$
\lim _{n \rightarrow \infty}\left\langle F_{n} \mid L F_{n}\right\rangle=\left\langle T_{t} F \mid L T_{t} F\right\rangle
$$

Take $F_{n}=T_{t, n} F$. Then, although $T_{t, n} F$ may not be in $\mathscr{D}_{0}$, there exist 
functions $G_{m} \in \mathscr{D}_{0}$ such that $G_{m} \rightarrow T_{t, n} F$ and $L G_{m} \rightarrow L T_{t, n} F$ as $m \rightarrow \infty$ in $\boldsymbol{L}^{2}\left(\boldsymbol{R}^{2 \alpha+1}, \mu^{(\alpha)}\right)$ and therefore in $\mathscr{H}$; remind Lemma $4.1(1)$. Thus, it is sufficient to show that

$$
\lim _{n \rightarrow \infty}\left\langle T_{t, n} F \mid L T_{t, n} F\right\rangle=\left\langle T_{t} F \mid L T_{t} F\right\rangle,
$$

since Lemma 5.3 proves $T_{t, n} F \rightarrow T_{t} F$ in $\mathscr{H}$. Noting that $L_{n} F=L F$ for $n$ large enough, we have

$$
\begin{aligned}
& \quad\left\langle T_{t, n} F \mid L T_{t, n} F\right\rangle-\left\langle T_{t} F \mid L T_{t} F\right\rangle|\leq|\left\langle T_{t, n} F \mid L T_{t, n} F-L_{n} T_{t, n} F\right\rangle \mid \\
& \quad+\left\|T_{t, n} L L_{n} F\right\|_{\mathscr{H}}\left\|T_{t, n} F-T_{t} F\right\|_{\mathscr{H}}+\left\|T_{t} F\right\|_{\mathscr{H}}\left\|T_{t, n} L F-T_{t} L F\right\|_{\mathscr{H}} .
\end{aligned}
$$

Here, the second and third terms tend to 0 as $n \rightarrow \infty$ by Lemma 5.2. For the first term, noting the facts:

$$
\begin{aligned}
\langle F \mid L G\rangle= & -\sum_{i \in \boldsymbol{Z}} \sum_{k \in \boldsymbol{Z}}\left\langle\nabla_{1, i} \frac{\partial \tau_{k} F}{\partial \sigma_{i}} \nabla_{1, i} \frac{\partial G}{\partial \sigma_{i}}\right\rangle, \\
\left\langle F \mid L_{n} G\right\rangle= & -\sum_{k \in \boldsymbol{Z}} \sum_{i=-n}^{n-1}\left\{\left\langle\nabla_{1, i} \frac{\partial \tau_{k} F_{1}}{\partial \sigma_{i}} \nabla_{1, i} \frac{\partial F_{2}}{\partial \sigma_{i}}\right\rangle\right. \\
& \left.+\left\langle\left(\frac{\partial \tau_{k} F}{\partial \sigma_{-n}}-\frac{\partial \tau_{k} F}{\partial \sigma_{n}}\right)\left(\frac{\partial G}{\partial \sigma_{-n}}-\frac{\partial G}{\partial \sigma_{n}}\right)\right\rangle\right\},
\end{aligned}
$$

we can use Lemma 5.6 to obtain

$$
\begin{aligned}
\left\langle\left\langle T_{t, n} F \mid L T_{t, n} F-L_{n} T_{t, n} F\right\rangle\right| & \mid-\sum_{k=1}^{2 n+1}\left\langle\tau_{k} \frac{\partial T_{t, n} F}{\partial \sigma_{n+1-k}} \frac{\partial T_{t, n} F}{\partial \sigma_{n}}\right\rangle+\sum_{k=-2 n}^{0}\left\langle\tau_{k} \frac{\partial T_{t, n} F}{\partial \sigma_{-n-k}} \frac{\partial T_{t, n} F}{\partial \sigma_{n}}\right\rangle \\
& +\sum_{k=0}^{2 n}\left\langle\tau_{k} \frac{\partial T_{t, n} F}{\partial \sigma_{n-k}} \frac{\partial T_{t, n} F}{\partial \sigma_{-n}}\right\rangle-\sum_{k=-2 n-1}^{-1}\left\langle\tau_{k} \frac{\partial T_{t, n} F}{\partial \sigma_{-n-1-k}} \frac{\partial T_{t, n} F}{\partial \sigma_{-n}}\right\rangle \mid \\
\leq & \left(\sum_{k=1}^{2 n+1}\left\langle\tau_{k}\left|\frac{\partial T_{t, n} F}{\partial \sigma_{n+1-k}}\right|\right\rangle+\sum_{k=-2 n}^{0}\left\langle\tau_{k}\left|\frac{\partial T_{t, n} F}{\partial \sigma_{-n-k}}\right|\right\rangle+\sum_{k=0}^{2 n}\left\langle\tau_{k}\left|\frac{\partial T_{t, n} F}{\partial \sigma_{n-k}}\right|\right\rangle\right. \\
& \left.+\sum_{k=-2 n-1}^{-1}\left\langle\tau_{k}\left|\frac{\partial T_{t, n} F}{\partial \sigma_{-n-1-k}}\right|\right\rangle\right) C_{F} e^{8(a+A) t} \frac{1}{(n-\alpha) !}(4(a+A) t)^{n-\alpha} \\
\leq & 8 e^{16(a+A) t}\left(\alpha-\frac{1}{2}+e^{4(a+A) t}\right) \frac{1}{(n-\alpha) !}(4(a+A) t)^{n-\alpha} .
\end{aligned}
$$

This tends to 0 as $n \rightarrow \infty$.

We conclude this paragraph by showing the following lemma which will be used in Section 6 .

Lemma 5.7. Let $F_{1} \in \mathscr{D}(L)$ and $F_{2} \in \mathscr{D}_{0} \cap \mathscr{D}\left(L_{n}\right)$. Then 


$$
\left\langle F_{1} \mid L_{n} F_{2}\right\rangle^{2} \leq C(n)\left\langle F_{1} \mid L F_{1}\right\rangle\left\langle F_{2} L_{n} F_{2}\right\rangle .
$$

where $C(n)=2(2 n+1)\left(4 n^{2}+2 n+1\right)$.

Proof. First, assume that $F_{1} \in \mathscr{D}_{0}$ with $\left\langle F_{1}\right\rangle=0$. Notice (5.19) and use the fact that for a sequence $\left\{a_{l}\right\}_{l \in Z}$,

$$
\sum_{l \in \boldsymbol{Z}} a_{l}=\sum_{l=-n}^{n} \sum_{m \in \boldsymbol{Z}} a_{l+(2 n+1) m}
$$

if $a_{l}=0$ for all $l \in Z$ but finite $l$ 's. Then some tedious but straightforward calculations prove that

$$
\frac{1}{2 n+1} \sum_{i \in \boldsymbol{Z}} \sum_{i \in \boldsymbol{Z}}\left\langle\sum_{i=-n}^{n} \nabla_{1, i} \frac{\partial \tau_{k} F_{1}}{\partial \sigma_{i}} \nabla_{1, i} \frac{\partial \tau_{l} F_{1}}{\partial \sigma_{i}}\right\rangle=-\left\langle F_{1} \mid L F_{1}\right\rangle .
$$

By (5.20) and (5.21) we have for

$$
\begin{aligned}
\left\langle F_{1} \mid L_{n} F_{2}\right\rangle^{2}= & \left(\sum_{k \in \boldsymbol{Z}} \sum_{i=-n}^{n}\left\langle\nabla_{1, i} \frac{\partial \tau_{k} F_{1}}{\partial \sigma_{i}} \nabla_{1, i}^{+} \frac{\partial F_{2}}{\partial \sigma_{i}}\right\rangle\right. \\
& \left.+\sum_{k \in \boldsymbol{Z}}\left\langle\left(\frac{\partial \tau_{k} F_{1}}{\partial \sigma_{-n}}-\frac{\partial \tau_{k} F_{1}}{\partial \sigma_{n+1}}\right)\left(\frac{\partial F_{2}}{\partial \sigma_{-n}}-\frac{\partial F_{2}}{\partial \sigma_{n}}\right)\right\rangle\right)^{2} \\
\leq & 2\left\langle\sum_{i=-n}^{n}\left(\sum_{k \in \boldsymbol{Z}} \nabla_{1, i} \frac{\partial \tau_{k} F_{1}}{\partial \sigma_{i}}\right) \nabla_{1, i}^{+} \frac{\partial F_{2}}{\partial \sigma_{i}}\right\rangle^{2} \\
& +2\left\langle\left(\sum_{i=-n}^{n} \sum_{k \in \boldsymbol{Z}} \nabla_{1, i} \frac{\partial \tau_{k} F_{1}}{\partial \sigma_{i}}\right)\left(\sum_{i=-n}^{n-1} \nabla_{1} \frac{\partial F_{2}}{\partial \sigma_{i}}\right)\right\rangle^{2} \\
\leq & 2 \sum_{k \in \boldsymbol{Z}} \sum_{i \in \boldsymbol{Z}} \sum_{i=-n}\left\langle\nabla_{1, i} \frac{\partial \tau_{k} F_{1}}{\partial \sigma_{i}} \nabla_{1, i} \frac{\partial \tau_{l} F_{1}}{\partial \sigma_{i}}\right\rangle \sum_{i=-n}^{n}\left\langle\left(\nabla_{1}^{+} \frac{\partial F_{2}}{\partial \sigma_{i}}\right)^{2}\right\rangle \\
& +4 n(2 n+1)\left\langle\sum_{i=-n}^{n}\left(\sum_{k \in \boldsymbol{Z}} \nabla_{1, i} \frac{\partial \tau_{k} F_{1}}{\partial \sigma_{i}}\right)^{2}\right\rangle\left\langle\sum_{i=-n}^{n-1}\left(\nabla_{1} \frac{\partial F_{2}}{\partial \sigma_{i}}\right)^{2}\right\rangle \\
\leq & 2(2 n+1)\left(4 n^{2}+2 n+1\right)\left\langle F_{1} \mid L F_{1}\right\rangle\left\langle F_{2} L_{n} F_{2}\right\rangle,
\end{aligned}
$$

where $\nabla_{1}^{+}$is defined by $\left(\nabla_{1}^{+} G\right)(i)=\nabla_{1} G(i),-n \leq i \leq n-1$ and $\left(\nabla_{1}^{+} G\right)(n)$ $=G(-n)-G(n)$. Consequently, the desired inequality is verified for $F_{1} \in \mathscr{D}_{0}$ and $F_{2} \in \mathscr{D}_{0} \cap \mathscr{D}\left(L_{n}\right)$. However, this concludes the proof with the help of Proposition 5.5.

\section{§6. Invariant subspace}

In this section, we show the ergodicity of $T_{t}$ in $\mathscr{H}$. Denote by $P \mathscr{H}$ the subspace of $\mathscr{H}$ invariant under $\left\{T_{t}\right\}$. Then the spectral theorem implies that

$$
\lim _{t \rightarrow \infty} T_{t} F=G \in P \mathscr{H}
$$


exists for every $F \in \mathscr{H}$. What we prove is that $P \mathscr{H}$ is one-dimensional subspace of $\mathscr{H}$. Let us denote the conditional expectation under $\mu_{\lambda}^{(n)}$ of $F \in \mathscr{F}_{2,[-n, n]}, n \in Z^{+}$on the hyperplane $\left\{\sigma \mid 1 /(2 n+1) \sum_{k=-n}^{n} \sigma_{k}=y\right\}$ by

$$
\nu_{y}^{(n)}(F)=\mu_{\lambda}^{(n)}\left(F \mid \frac{1}{2 n+1} \sum_{k=-n}^{n} \sigma_{k}=y\right), \quad y \in \boldsymbol{R},
$$

and

$$
\left(\Gamma_{n} F\right)(\sigma)=\nu_{1 /(2 n+1)}^{(n)} \Sigma_{k=-n^{\sigma_{k}}}^{n}(F) .
$$

Note that $\nu_{y}^{(n)}$ is determined independently of $\lambda$.

First, we show the following property of $P \mathscr{H}$ :

Proposition 6.1. Let $G \in P \mathscr{H}$. Then for every $F \in \mathscr{D}_{0} \cap \mathscr{F}_{2,[-n, n]}$

$$
\left\langle G \mid \Gamma_{n} F\right\rangle=\langle G \mid F\rangle \text {. }
$$

Proof. Proposition 5.3 verifies $G \in \mathscr{D}(L)$ and $L G=s-\lim _{t \rightarrow 0} \frac{1}{t}\left(T_{t} G-G\right)$ $=0$ in $\mathscr{H}$. Moreover by Lemma 5.7

$$
\left\langle G \mid L_{n} F\right\rangle^{2} \leq C(n)\langle G \mid L G\rangle\left\langle F L_{n} F\right\rangle \quad \text { for } F \in \mathscr{D}_{0} \cap \mathscr{D}\left(L_{n}\right),
$$

and therefore

$$
\left\langle G \mid L_{n} F\right\rangle=0 \quad \text { for each } F \in \mathscr{D}_{0} \cap \mathscr{D}\left(L_{n}\right) .
$$

For every $F \in \mathscr{D}_{0} \cap \mathscr{F}_{2,[-n, n]}$, noting $T_{t, n} F \in \mathscr{D}\left(L_{n}\right)$ for $t \geq 0$, we choose $F_{m} \in \mathscr{D}_{0} \cap \mathscr{D}\left(L_{n}\right)$ such that $F_{m} \rightarrow T_{t, n} F$ and $L_{n} F_{m} \rightarrow L_{n} T_{t, n} F$ as $m \rightarrow \infty$ in $L^{2}\left(\boldsymbol{R}^{2 n+1}, \mu_{\lambda}^{(n)}\right)$. This is actually possible because $\mathscr{D}_{0} \cap \mathscr{D}\left(L_{n}\right)$ is a core for $L_{n}$ in $L_{i}^{2}\left(R^{2 n+1}, \mu_{\lambda}^{(n)}\right)$. However, Lemma 4.1(1) proves that $F_{m} \rightarrow T_{t, n} F$ and $L_{n} F_{m} \rightarrow L_{n} T_{t, n} F$ as $m \rightarrow \infty$ also in $\mathscr{H}$. Hence, from (6.6), we have

$$
\left\langle G \mid L_{n} T_{t, n} F\right\rangle=0 \quad \text { for each } t \geq 0 \text { and } F \in \mathscr{D}_{0} .
$$

Lemma 4.1(1) verifies also that $T_{t, n} F$ is strongly differentiable in $\mathscr{H}$ as well as in $L^{2}\left(R^{2 n+1}, \mu_{\lambda}^{(n)}\right)$ and $(d / d t) T_{t, n} F=L_{n} T_{t, n} F$. We therefore have

$$
\frac{d}{d t}\left\langle G \mid T_{t, n} F\right\rangle=0 \quad \text { for each } t \geq 0 \text { and } F \in \mathscr{D}_{0} \text {. }
$$

This implies

$$
\left\langle G \mid T_{t, n} F\right\rangle=\langle G \mid F\rangle \quad \text { for each } t \geq 0 .
$$

Since the diffusion process with generator $L_{n}$ is ergodic on every hyperplane $\left\{\sigma \mid 1 /(2 n+1) \sum_{k=-n}^{n} \sigma_{k}=y\right\}$, we have for each $F \in \mathscr{D}_{0}$ 


$$
\lim _{t \rightarrow \infty} T_{t, n} F=\Gamma_{n} F
$$

strongly in $L^{2}\left(\boldsymbol{R}^{2 n+1}, \mu_{\lambda}^{(n)}\right)$. Lemma 4.1(1) again implies that (6.10) holds in $\mathscr{H}$. Letting $t \rightarrow \infty$ in (6.9) establishes the conclusion.

For $g \in C_{0}^{\infty}(R)$ such that $\int g(x) d x=1$, we define

$$
F_{0}(g)(\sigma)=\int\left(\sigma_{[x]}-\rho^{\prime}(\lambda)\right) g(x) d x .
$$

Remark. (1) The definition of $F_{0}(g)$ is independent of the choice of $g$, i.e. for $g_{1}, g_{2} \in C_{0}^{\infty}(R)$ such that $\int g_{1}(x) d x=\int g_{2}(x) d x=1, F_{0}\left(g_{1}\right)=F_{0}\left(g_{2}\right)$ in $\mathscr{H}$.

(2) Particularly, we can take $g=1 /(2 n+1) \chi_{[-n, n]}$ in (6.11), although this $g$ is not in $C_{0}^{\infty}(\boldsymbol{R})$. We therefore have

$$
F_{0}(g)=\frac{1}{2 n+1} \sum_{k=-n}^{n}\left(\sigma_{k}-\rho^{\prime}(\lambda)\right) \quad \text { in } \mathscr{H}
$$

for each $n \in Z^{+}$and $g \in C_{0}^{\infty}(\boldsymbol{R})$ satisfying $\int g(x) d x=1$.

The purpose of this section is to show the following.

Proposition 6.2. Let $G \in P \mathscr{H}$. Then

$$
G=\rho^{\prime \prime}(\lambda)^{-1}\left\langle G \mid F_{0}(g)\right\rangle F_{0}(g) .
$$

Proof. For every $F \in \mathscr{D}_{0} \cap \mathscr{F}_{2,[-n, n]}$, we have by Proposition 6.1

$$
\left\langle G \mid \Gamma_{n} F\right\rangle=\langle G \mid F\rangle
$$

and, therefore, by (6.12) and Lemma 6.3 which will be stated later

$$
\begin{aligned}
\langle G \mid F\rangle & =\lim _{n \rightarrow \infty}\left\langle G \mid \Gamma_{n} F\right\rangle \\
& =\lim _{n \rightarrow \infty}\left\langle G \mid \rho^{\prime \prime}(\lambda)^{-1} \frac{d}{d \lambda}\langle F\rangle_{\lambda}\left(\frac{1}{2 n+1} \sum_{k=-n}^{n} \sigma_{k}-\rho^{\prime}(\lambda)\right)\right\rangle \\
& =\rho^{\prime \prime}(\lambda)^{-1} \frac{d}{d \lambda}\langle F\rangle_{\lambda}\left\langle G \mid F_{0}(g)\right\rangle .
\end{aligned}
$$

Here we remind the notation: $[\cdot]_{\lambda}=\boldsymbol{E}^{\mu_{\lambda}}[\cdot]$. It is, however, easy to check that

$$
\frac{d}{d \lambda}\langle F\rangle_{\lambda}=\left\langle F \mid F_{0}(g)\right\rangle \quad \text { for every } F \in \mathscr{D}_{0} \text {. }
$$

Combining (6.14) nad (6.15), we obtain 


$$
\langle G \mid F\rangle=\rho^{\prime \prime}(\lambda)^{-1}\left\langle F \mid F_{0}(g)\right\rangle\left\langle G \mid F_{0}(g)\right\rangle \text {. }
$$

Consequently,

$$
\left\langle G-\rho^{\prime \prime}(\lambda)^{-1}\left\langle G \mid F_{0}(g)\right\rangle F_{0}(g) \mid F\right\rangle=0 \quad \text { for all } F \in \mathscr{D}_{0} .
$$

Since $\mathscr{D}_{0}$ is dense in $\mathscr{H}$, we have the conclusion.

We have used the following lemma for the proof of Proposition 6.2.

Lemma 6.3. Let $F \in \mathscr{D}_{0}$. Then

$$
\lim _{n \rightarrow \infty}\left\|\Gamma_{n} F-\rho^{\prime \prime}(\lambda)^{-1} \frac{d}{d \lambda}\langle F\rangle_{\lambda}\left(\frac{1}{2 n+1} \sum_{k=-n}^{n} \sigma_{k}-\rho^{\prime}(\lambda)\right)\right\|_{\mathscr{x}}=0 .
$$

Proof. By Lemma 4.2, it is sufficient to show that

$$
\lim _{n \rightarrow \infty} n\left\{I_{1}(n)+I_{2}(n)\right\}=0
$$

where

$$
\begin{aligned}
I_{1}(n)=2 & \left\langle\left(\Gamma_{n} F-\langle F\rangle_{h^{\prime}(1 /(2 n+1)} \Sigma_{k=-n^{\sigma_{k}}}^{n}\right)^{2}\right\rangle \\
I_{2}(n)=2 & \left\langle\left(\langle F\rangle_{h^{\prime}(1 /(2 n+1)} \Sigma_{k=-n^{\left.\sigma_{k}\right)}}^{n}-\langle F\rangle_{\lambda}\right.\right. \\
& \left.\left.-\rho^{\prime \prime}(\lambda)^{-1} \frac{d}{d \lambda}\langle F\rangle_{\lambda}\left(\frac{1}{2 n+1} \sum_{k=-n}^{n} \sigma_{k}-\rho^{\prime}(\lambda)\right)\right)^{2}\right\rangle .
\end{aligned}
$$

It will be shown later in Lemmas 6.4 and 6.6 that both $n \cdot I_{1}(n)$ and $n \cdot I_{2}(n)$ tend to zero as $n \rightarrow \infty$.

Lemma 6.4. Let $F \in \mathscr{D}_{0}$. Then

$$
\lim _{n \rightarrow \infty} n\left\langle\left(\Gamma_{n} F-\langle F\rangle_{h^{\prime}(1 /(2 n+1)} \Sigma_{k=-n^{\sigma_{k}}}^{n}\right)^{2}\right\rangle=0 .
$$

In order to prove this lemma, we use the following local central limit theorem:

Lemma 6.5. For $\eta \in \boldsymbol{R}$, let $\left\{X_{n}\right\}$ be a sequence of $\boldsymbol{R}$-valved independent random variables with the same distribution $q_{\eta}\left(x+\rho^{\prime}(\eta)\right) d x$. Let $f_{n}(x, \eta)$ be the density function of $1 / \sqrt{ } \bar{n} \sum_{k=1}^{n} X_{k}$. Then, for $\lambda \in \boldsymbol{R}$, there exists $\varepsilon_{0}>0$ such that

$$
f_{n}(x, \eta)=\left(2 \pi \rho^{\prime \prime}(\eta)\right)^{-1 / 2} \exp \left[-\frac{x^{2}}{2 \rho^{\prime \prime}(\eta)}\right]+r_{1}(x, \eta) n^{-1 / 2}+o\left(n^{-1 / 2}\right)
$$

uniformly in $x \in R$ and $\eta \in\left[\lambda-\varepsilon_{0}, \lambda+\varepsilon_{0}\right]$, where 


$$
\begin{aligned}
r_{1}(x, \eta) & =6^{-1}(2 \pi)^{-1 / 2} \rho^{\prime \prime}(\eta)^{-7 / 2} M_{3}(\eta)\left(x^{3}-3 \rho^{\prime \prime}(\eta) x\right) \exp \left[-\frac{x^{2}}{2 \rho^{\prime \prime}(\eta)}\right], \\
M_{3}(\eta) & =\int x^{3} q_{\eta}\left(x+\rho^{\prime}(\eta)\right) d x .
\end{aligned}
$$

Proof. The proof is essentially given in Petrov [5]. The only different point is that, in our case, we need to check the uniformity in $\eta$. But, since $\rho$ and $h$ are smooth functions, one can do it easily.

We notice the following fact:

$$
\left\langle\left(\frac{1}{2 n+1} \sum_{k=-n}^{n} \sigma_{k}-\rho^{\prime}(\lambda)\right)^{4}\right\rangle_{2} \sim O\left(n^{-2}\right)
$$

wichh can be established by a direct computation and will be useful for the proofs of Lemmas 6.4 and 6.6.

Proof of Lemma 6.4. We assume that $F=F\left(\sigma_{-\alpha}, \cdots, \sigma_{\alpha}\right)$.

Step 1. We compute for $n \geq \alpha+1$

$$
\begin{aligned}
\nu_{y}^{(n)}(F)= & \mu_{\lambda}^{(n)}\left(F \mid \frac{1}{2 n+1} \sum_{k=-n}^{n} \sigma_{k}=y\right) \\
= & \hat{Z}(n, y)^{-1} \int_{R^{2 n}} d \sigma_{-n} \cdots d \sigma_{n-1} F\left(\sigma_{-\alpha}, \cdots, \sigma_{\alpha}\right) . \\
& \quad \times \exp \Psi\left((2 n+1) y, y, \sigma_{[-n, n-1]}\right) \\
= & \hat{Z}(n, y)^{-1} M\left(h^{\prime}(y)\right)^{-2 \alpha-1} \int_{R^{2 \alpha+1}} d \sigma_{-\alpha} \cdots d \sigma_{\alpha} F\left(\sigma_{-\alpha}, \cdots, \sigma_{\alpha}\right) . \\
& \quad \times \exp \left[h^{\prime}(y) \sum_{k=-\alpha}^{\alpha} \sigma_{k}-\sum_{k=-\alpha}^{\alpha} U\left(\sigma_{k}\right)\right] \cdot I_{n, y}\left(\sigma_{-\alpha}, \cdots, \sigma_{\alpha}\right) \\
= & \hat{Z}(n, y)^{-1}\left\langle F I_{n, y}\right\rangle_{h^{\prime}(y)}
\end{aligned}
$$

where

$$
\Psi\left(x, y, \sigma_{A}\right)=x h^{\prime}(y)-\sum_{k \in A \cap Z} U\left(\sigma_{k}\right)-U\left(x-\sum_{k \in A \cap Z} \sigma_{k}\right),
$$

for $x, y \in \boldsymbol{R}$ and $\sigma_{A}=\left\{\sigma_{k} ; k \in A \cap Z\right\}$, and

$$
\begin{aligned}
& \hat{Z}(n, y)= \int_{R^{2 n}} d \sigma_{-n} \cdots d \sigma_{n-1} \exp \Psi\left((2 n+1) y, y, \sigma_{[-n, n-1]}\right) \\
& I_{n, y}=M\left(h^{\prime}(y)\right)^{2 \alpha+1} \int_{R^{2 n-2 \alpha-1}} d \sigma_{-n} \cdots d \sigma_{-\alpha-1} d \sigma_{a+1} \cdots d \sigma_{n-1} . \\
& \quad \times \exp \Psi\left((2 n+1) y-\sum_{k=-\alpha}^{\alpha} \sigma_{k}, y, \sigma_{[-n,-\alpha-1] \cup[\alpha+1, n-1]}\right) .
\end{aligned}
$$


Let $f_{n}(x, \lambda)$ be the function defined in Lemma 6.5 and put $\eta=h^{\prime}(y)$. Then it is easy to see by a simple computation

$$
f_{n}(x, \eta)=\sqrt{n} M(\eta)^{-n} Z\left(n, \sqrt{n} x+n \rho^{\prime}(\eta)\right) \exp \left[\eta\left(\sqrt{n} x+\rho^{\prime}(\eta)\right]\right.
$$

where

$$
Z(n, y)=\int_{R^{n-1}} d \sigma_{1} \cdots d \sigma_{n-1} \exp \left[-\sum_{k=1}^{n-1} U\left(\sigma_{k}\right)-U\left(y-\sum_{k=1}^{n-1} \sigma_{k}\right)\right] .
$$

This implies

$$
Z(n, \sqrt{n} x+n y)=\sqrt{n} M(\eta)^{n} e^{-\eta(\sqrt{x} n+n y)} f_{n}(x, \eta) .
$$

Consequently, by (6.24), (6.27) and (6.28)

$$
\begin{aligned}
\hat{Z}(n, y) & =e^{\eta(2 n+1) y} Z(2 n+1,(2 n+1) y) \\
& =(2 n+1)^{-1 / 2} M(\eta)^{2 n+1} f_{2+1}(0, \eta),
\end{aligned}
$$

and by (6.25), (6.27) and (6.28)

$$
\begin{aligned}
I_{n, y} & =M(\eta)^{2 \alpha+1} Z\left(2(n-\alpha),(2 n+1) y-\sum_{k=-\alpha}^{\alpha} \sigma_{k}\right) \exp \left[\eta\left((2 n+1) y-\sum_{k=-\alpha}^{\alpha} \sigma_{k}\right)\right] \\
& =M(\eta)^{2 n+1}(2(n-\alpha))^{-1 / 2} f_{2(n-\alpha)}\left(\frac{2 \alpha+1}{\sqrt{2(n-\alpha)}}\left(y-\frac{1}{2 \alpha+1} \sum_{k=-\alpha}^{\alpha} \alpha_{k}\right), \eta\right) .
\end{aligned}
$$

Take $\varepsilon_{0}$ as in Lemma 6.5. Then, by the continuity of $y \rightarrow \eta=h^{\prime}(y)$, there exists $\delta_{0}>0$ such that

$$
|\eta-\lambda|=\left|h^{\prime}(y)-\lambda\right| \leq \varepsilon_{0}
$$

for every $y:\left|y-\rho^{\prime}(\lambda)\right| \leq \delta_{0}$. We set

$$
y_{n, \alpha}=\frac{2 \alpha+1}{\sqrt{2(n-\alpha)}}\left(y-\frac{1}{2 \alpha+1} \sum_{k=-\alpha}^{\alpha} \alpha_{k}\right) .
$$

By $(6.29) \sim(6.31)$ and Lemma 6.5, we have

$$
\begin{aligned}
\hat{Z}(n, y)^{-1} I_{n, y}{ }^{-1}= & \left(\frac{2 n+1}{2(n-\alpha)}\right)^{1 / 2} f_{2(n-\alpha)}\left(y_{n, \alpha}, \eta\right) f_{2 n+1}^{-1}(0, \eta)-1 \\
= & \left(1+o\left(n^{-1 / 2}\right)\right)\{1 \\
& \left.+J_{n, y}+\rho^{\prime \prime}(\eta)^{1 / 2} o\left(n^{-1 / 2}\right)\right\} \\
& \times\left\{1+\rho^{\prime \prime}(\eta)^{1 / 2} o\left(n^{-1 / 2)}\right)\right\}^{-1}-1
\end{aligned}
$$

uniformly in $\left(\sigma_{-\alpha}, \cdots, \sigma_{\alpha}\right) \in \boldsymbol{R}^{2 \alpha+1}$ and $y \in\left[\rho^{\prime}(\lambda)-\delta_{0}, \rho^{\prime}(\lambda)+\delta_{0}\right]$, where we denote

$$
J_{n, y}=\exp \left[-\frac{y_{n, \alpha}^{2}}{2 \rho^{\prime \prime}(\eta)}\right]-1+\left(2 \pi \rho^{\prime \prime}(\eta)\right)^{1 / 2} r_{1}\left(y_{n, \alpha}, \eta\right)(2(n-\alpha))^{-1 / 2},
$$


Since $\rho^{\prime \prime}(\eta)^{1 / 2}$ is bounded on $\left[\lambda-\varepsilon_{0}, \lambda+\varepsilon_{0}\right]$,

$$
\rho^{\prime \prime}(\eta)^{1 / 2} o\left(n^{-1 / 2}\right)=o\left(n^{-1 / 2}\right) .
$$

Combining (6.32) and (6.34),

$$
\hat{Z}(n, y)^{-1} I_{n, y}-1=J_{n, y}+J_{n, y} o\left(n^{-1 / 2}\right)+o\left(n^{-1 / 2}\right) .
$$

Consequently,

(6.35) $\left\langle\left(\hat{Z}(n, y)^{-1} I_{n, y}-1\right)^{2}\right\rangle_{h^{\prime}(y)} \leq 3\left\langle J_{n, y}^{2}\right\rangle_{h^{\prime}(y)}+\left\langle J_{n, y}^{2}\right\rangle_{h^{\prime}(y)} o\left(n^{-1}\right)+o\left(n^{-1}\right)$.

By (6.20) and (6.31), we have

$$
\begin{aligned}
J_{n, y}= & -\frac{y_{n, \alpha}^{2}}{2 \rho^{\prime \prime}(\eta)} e^{-\theta} \\
& +\frac{1}{6} \rho^{\prime \prime}(\eta)^{-3} M_{3}(\eta)\left(y_{n, \alpha}^{3}-3 \rho^{\prime \prime}(\eta) y_{n, \alpha}\right) \exp \left[-\frac{y_{n, \alpha}^{2}}{2 \rho^{\prime \prime}(\eta)}\right](2(n-\alpha))^{-1 / 2} \\
= & \left\{\left(y-\frac{1}{2 \alpha+1} \sum_{k=-\alpha}^{\alpha} \sigma_{k}\right)^{2} e^{-\theta}+\left(y-\frac{1}{2 \alpha+1} \sum_{k=-\alpha}^{\alpha} \sigma_{k}\right) .\right. \\
& \left.\times\left(\frac{(2 \alpha+1)^{2}}{2(n-\alpha)}\left\{y-\frac{1}{2 \alpha+1} \sum_{k=-\alpha}^{\alpha} \sigma_{k}\right\}^{2}-3 \rho^{\prime \prime}(\eta)\right) \exp \left[-\frac{y_{n, \alpha}^{2}}{2 \rho^{\prime \prime}(\eta)}\right]\right\} o\left(n^{-1 / 2}\right)
\end{aligned}
$$

with some $\theta \in\left(0, y_{n, \alpha}^{2} / 2 \rho^{\prime \prime}(\eta)\right)$. Set

$$
\hat{J}_{y}=\left(y-\frac{1}{2 \alpha+1} \sum_{k=-\alpha}^{\alpha} \sigma_{k}\right)^{2}\left\{1+\left(y-\frac{1}{2 \alpha+1} \sum_{k=-\alpha}^{\alpha} \sigma_{k}\right)^{4}\right\} .
$$

Then

$$
\left|J_{n, y}\right|^{2} \leq \hat{J}_{y} o\left(n^{-1}\right)
$$

However it is easy to see that $y \rightarrow\left\langle\hat{J}_{y}\right\rangle_{h^{\prime}(y)}$ is continuous and therefore

$$
\begin{aligned}
& \left\langle J_{n, y}^{2}\right\rangle_{n^{\prime}(y)} \leq\left\langle\hat{J}_{y}\right\rangle_{n^{\prime}(y)} o\left(n^{-1}\right)=o\left(n^{-1}\right) \\
& \text { uniformly in } y \in\left[\rho^{\prime}(\lambda)-\delta_{0}, \rho^{\prime}(\lambda)+\delta_{0}\right] .
\end{aligned}
$$

Combining (6.35) and (6.37), we have

$$
\begin{aligned}
& \left\langle\left(\hat{Z}(n, y)^{-1} I_{n, y}-1\right)^{2}\right\rangle_{h^{\prime}(y)}=o\left(n^{-1}\right) \\
& \text { uniformly in } y \in\left[\rho^{\prime}(\lambda)-\delta_{0}, \rho^{\prime}(\lambda)+\delta_{0}\right] .
\end{aligned}
$$

Step 2. Set

$$
A_{n}=\left\{\sigma \in \boldsymbol{R}^{Z}:\left|\frac{1}{2 n+1} \sum_{k=-n}^{n} \sigma_{k}-\rho^{\prime}(\lambda)\right|>\delta_{0}\right\}
$$

By (6.23) and (6.38) 


$$
\begin{aligned}
\left\langle\left(\Gamma_{n} F\right.\right. & \left.\left.-\langle F\rangle_{h^{\prime}(1 /(2 n+1)} \Sigma_{\left.k=-n^{\sigma_{k}}\right)}^{n}\right)^{2} ; A_{n}^{c}\right\rangle \\
& =\left\langle\left.\left(\left\langle\hat{Z}(n, y)^{-1} F I_{n, y}-F\right\rangle_{h^{\prime}(y)}\right)^{2}\right|_{y=1 /(2 n+1)} \Sigma_{k=-n^{\sigma_{k}}}^{n} ; A_{n}^{c}\right\rangle \\
& \left.\leq\left.\|F\|_{\infty}^{2}\left\langle\left\langle(\hat{Z} n, y)^{-1} I_{n, y}-1\right)^{2}\right\rangle_{h^{\prime}(y)}\right|_{y=1 /(2 n+1)} \Sigma_{k=-n^{\sigma_{k}}}^{n} ; A_{n}^{c}\right\rangle \\
& =o\left(n^{-1}\right) \quad \text { as } n \rightarrow \infty .
\end{aligned}
$$

On the other hand, by (6.22)

$$
\begin{aligned}
& \left\langle\left(\Gamma_{n} F-\langle F\rangle_{n^{\prime}(1 /(2 n+1)} \Sigma_{\left.k=-n^{\sigma_{k}}\right)}^{n}\right)^{2} ; A_{n}\right\rangle \leq 4\|F\|_{\infty}^{2} \mu_{\lambda}\left(A_{n}\right) \\
& \leq 4\|F\|_{\infty}^{2} \delta_{0}^{4}\left\langle\left(\frac{1}{2 n+1} \sum_{k=-n}^{n} \sigma_{k}-\rho^{\prime}(\lambda)\right)^{4}\right\rangle=o\left(n^{-1}\right) \quad \text { as } n \rightarrow \infty .
\end{aligned}
$$

The combination of (6.40) and (6.41) proves the conclusion.

Lemma 6.6. Let $F \in \mathscr{D}_{0}$. Then

$$
\begin{aligned}
\lim _{n \rightarrow \infty} n\left\langle\left\{\langle F\rangle_{h^{\prime}(1 /(2 n+1)} \Sigma_{k=-n^{\left.\sigma_{k}\right)}}^{n}-\langle F\rangle_{2}\right.\right. \\
\left.\left.-\quad \rho^{\prime \prime}(\lambda)^{-1} \frac{d}{d \lambda}\langle F\rangle_{\lambda}\left(\frac{1}{2 n+1} \sum_{k=-}^{n} \sigma_{k}-\rho^{\prime}(\lambda)\right)\right\}^{2}\right\rangle=0 .
\end{aligned}
$$

Proof. Set

$$
J_{n}(y)=\langle F\rangle_{h^{\prime}(y)}-\langle F\rangle_{\lambda}-\rho^{\prime \prime}(\lambda)^{-1} \frac{d}{d \lambda}\langle F\rangle_{2}\left(y-\rho^{\prime}(\lambda)\right) .
$$

Then, the conclusion follows if we show that

$$
\left\langle J_{n}\left(\frac{1}{2 n+1} \sum_{k=-n}^{n} \sigma_{k}\right)^{2}\right\rangle=o\left(n^{-1}\right) \quad \text { as } n \rightarrow \infty .
$$

Let $A_{n}$ be the set defined by (6.39). Then, we have by (6.15) and (6.22)

$$
\begin{aligned}
& \left\langle J_{n}\left(\frac{1}{2 n+1} \sum_{k=-n}^{n} \sigma_{k}\right)^{2} ; A_{n}\right\rangle \\
& \left.\quad \leq 6\|F\|_{\infty}^{2}\left(\delta_{0}^{4}+3(2 \alpha+1)\right)^{2} \delta_{0}^{2}\right)\left\langle\left(\frac{1}{2 n+1} \sum_{k=-n}^{n} \sigma_{k}-\rho^{\prime}(\lambda)\right)^{4}\right\rangle \\
& \quad=o\left(n^{-1}\right) .
\end{aligned}
$$

Noting that

$$
\left.\frac{d}{d y}\langle F\rangle_{h^{\prime}(y)}\right|_{y=\rho^{\prime}(\lambda)}=\rho^{\prime \prime}(\lambda)^{-1} \frac{d}{d \lambda}\langle\dot{F}\rangle_{\lambda}
$$

we have

$$
J_{n}(y)=\left.\frac{1}{2} \frac{d^{2}}{d y^{2}}\langle F\rangle_{h^{\prime}(y)}\right|_{y=\theta}\left(y-\rho^{\prime}(\lambda)\right)^{2}
$$


with some $\theta \in\left(\rho^{\prime}(\lambda) \wedge y, \rho^{\prime}(\lambda) \vee y\right)$. Notice that $\left|y-\rho^{\prime}(\lambda)\right| \leq \delta_{0}$ implies $\left|\theta-\rho^{\prime}(\lambda)\right| \leq \delta_{0}$. Since the function $y \rightarrow\langle F\rangle_{h^{\prime}(y)}$ belongs to $\boldsymbol{C}^{2}(\boldsymbol{R})$, there exists $C>0$ such that

$$
\left|\frac{d^{2}}{d y^{2}}\langle F\rangle_{h^{\prime}(y)}\right| \leq C \quad \text { for } y \in\left[\rho^{\prime}(\lambda)-\delta_{0}, \rho^{\prime}(\lambda)+\delta_{0}\right] .
$$

By combining (6.46) with (6.47), and using (6.22), we obtain

$$
\begin{aligned}
\left\langle J_{n}\left(\frac{1}{2 n+1} \sum_{k=-n}^{n} \sigma_{k}\right)^{2} ; A_{n}^{c}\right\rangle & \leq \frac{C}{2}\left\langle\left(\frac{1}{2 n+1} \sum_{k=-n}^{n} \sigma_{k}-\rho^{\prime}(\lambda)\right)^{4}\right\rangle \\
& =o\left(n^{-1}\right) .
\end{aligned}
$$

This establishes (6.44) with the help of (6.45).

§7. Tightness of $\left\{P_{\varepsilon}: 0<\varepsilon \leq 1\right\}$

The Boltzmann-Gibbs principle has been established by combining the results of Sections 4,5 and 6 . In order to show the tightness of $\left\{P_{\varepsilon}: 0<\varepsilon \leq 1\right\}$ being defined in Section 2, we first derive the following estimate. The duality between two spaces $\mathscr{E}_{r}^{\prime}$ and $\mathscr{E}_{r}$ will be simply denoted by $($,$) .$

Lemma 7.1. For $f \in \mathscr{E}_{r}$ and $F \in L^{4}\left(\boldsymbol{R}, q_{\lambda}(x) d x\right)$ satisfying $\int_{\boldsymbol{R}} F(x) q_{\lambda}(x) d x$ $=0$, there exists a constant $C=C(F, f)>0$ such that

$$
\left\langle\left(F\left(\sigma_{[x / \varepsilon]}\right), \quad D_{\varepsilon}^{k} f(x)\right)^{4}\right\rangle_{x} \leq C \varepsilon^{2}, \quad k=0,1,2
$$

where $D_{\varepsilon}^{0} f=f, D_{\varepsilon}^{1} f(x)=\nabla_{\varepsilon}^{*} f(x)=\varepsilon^{-1}(f(x-\varepsilon)-f(x))$ and $D_{\varepsilon}^{2} f=\Delta_{\varepsilon} f$.

Proof. Set $g=D_{\varepsilon}^{k} f, k=0,1,2$. Noting that $\left\langle F\left(\sigma_{i}\right)\right\rangle=0$ for $i \in Z$, we have

$$
\begin{aligned}
& \left\langle\left(F\left(\sigma_{[x / \varepsilon]}\right), g(x)\right)^{4}\right\rangle_{2}=\left\langle\left(\sum_{i=-\infty}^{\infty} F\left(\sigma_{i}\right) \int_{\epsilon i}^{\epsilon(i+1)} g(x) d x\right)^{4}\right\rangle \\
& =\left\langle\sum_{i=-\infty}^{\infty}\left(F\left(\sigma_{i}\right) \int_{\varepsilon i}^{\epsilon(i+1)} g(x) d x\right)^{4}\right\rangle \\
& \quad+6\left\langle\sum_{i=-\infty}^{\infty} \sum_{j=-\infty}^{i-1}\left(F\left(\sigma_{i}\right) \int_{\varepsilon i}^{s(i+1)} g(x) d x\right)^{2}\left(F\left(\sigma_{j}\right) \int_{\varepsilon j}^{\varepsilon(j+1)} g(x) d x\right)^{2}\right\rangle \\
& \leq 7\|F\|_{L_{4}\left(q_{\lambda}\right)}^{4}\|g\|_{4}^{4} \varepsilon^{2} .
\end{aligned}
$$

Since $f \in \mathscr{E}_{r}$ implies $\sup _{0<\varepsilon \leq 1, k}\left\|D_{\varepsilon}^{k} f\right\|_{4}=C_{f}<\infty$, (7.2) proves the conclusion with $C=7 C_{f}^{4}\|F\|_{\boldsymbol{L}_{4}\left(q_{\lambda}\right)}^{4}$.

Proposition 7.2. $\left\{P_{\varepsilon}: 0<\varepsilon \leq 1\right\}$ is tight on $\mathscr{C}$. 
Proof. Since $\mathscr{E}_{r}$ is a nuclear Fréchet space, by the theorem in [4], $\left\{P_{\varepsilon}: 0<\varepsilon \leq 1\right\}$ is tight on $\mathscr{C}$ if the family of distribution on $C([0, \infty) ; \boldsymbol{R})$ of $V_{t}^{\epsilon}(f)=\int V_{\varepsilon}(t, x) f(x) d x: 0<\varepsilon \leq 1$, is tight for each $f \in \mathscr{E}_{r}$. However, Lemma 7.1 implies $\boldsymbol{E}\left[V_{0}^{\varepsilon}(f)^{4}\right] \leq C$ for $\varepsilon \in(0,1]$ with some $C>0$. Therefore, noting the stationarity of $V_{t}^{\varepsilon}(f)$, it is sufficient for us to show that there exists constant $M>0$ such that

$$
E\left[\left(V_{t}^{\varepsilon}(f)-V_{0}^{s}(f)\right)^{4}\right] \leq M t^{3 / 2}, \quad \text { for } t \in[0,1] .
$$

Set $I(t)=E\left[\left(S_{t}^{s}(f)-S_{0}^{\varepsilon}(f)\right)^{4}\right]$, where $S_{t}^{\varepsilon}(f)=\int S_{\varepsilon}(t, x) f(x) d x$. Then, by Itô's formula and Hölder's inequality

$$
\begin{aligned}
I(t)= & 4 \int_{0}^{t} d s E\left[\left(S_{s}^{\varepsilon}(f)-S_{0}^{\varepsilon}(f)\right)^{3}\left(U^{\prime}\left(S_{\varepsilon}(s, x)\right), \Delta_{\varepsilon} f\right)\right] \\
& +12 \int_{0}^{t} d s \sum_{k=-\infty}^{\infty}\left(\int_{s k}^{s(k+1)} \nabla_{\varepsilon}^{*} f(x) d x\right)^{2} E\left[\left(S_{s}^{\varepsilon}(f)-S_{0}^{\varepsilon}(f)\right)^{2}\right] \\
\leq & 4 \int_{0}^{t} d s I(s)^{3 / 4} I_{1}(s)^{1 / 4}+12 \varepsilon \int_{0}^{t} d s\left\|\nabla_{\varepsilon}^{*} f\right\|^{2} I(s)^{1 / 2}
\end{aligned}
$$

where

$$
I_{1}(s)=E\left[\left(U^{\prime}\left(S_{\varepsilon}(s, x)\right), \Delta_{\varepsilon} f\right)^{4}\right]=\left\langle\left(U^{\prime}\left(\sigma_{[x / \varepsilon]}\right), \Delta_{\varepsilon} f\right)^{4}\right\rangle_{\lambda} .
$$

Notice that

$$
\begin{aligned}
I(s) & \leq 8 \boldsymbol{E}\left[\left(S_{s}^{\varepsilon}(f)-\rho^{\prime}(\lambda)^{4}\right]+8 \boldsymbol{E}\left[\left(S_{0}^{e}(f)-\rho^{\prime}(\lambda)\right)^{4}\right]\right. \\
& =16\left\langle\left(\sigma_{[x / \varepsilon]}-\rho^{\prime}(\lambda), f(x)\right)^{4}\right\rangle .
\end{aligned}
$$

Since $\int\left(U^{\prime}(x)-\lambda\right) q_{2}(x) d x=0$ and $\int\left(x-\rho^{\prime}(\lambda)\right) q_{\lambda}(x) d x=0$, by Lemma 7.1 , there exist $C_{0}$ and $C_{1}>0$ independent of $\varepsilon$ such that

$$
I(s) \leq C_{0} \varepsilon^{2},
$$

and

$$
I_{1}(s) \leq C_{1} \varepsilon^{2}, \quad \text { for } 0<\varepsilon \leq 1 .
$$

Moreover, from the proof of Lemma 7.1, we know that

$$
\left\|\nabla_{\varepsilon}^{*} f\right\| \leq C_{f}, \quad \text { for } 0<\varepsilon \leq 1 .
$$

From (7.4) (7.7), we have

$$
I(t) \leq C^{\prime} t \varepsilon^{2}
$$

where $C^{\prime}=4 C_{0}^{3 / 4} C_{1}^{1 / 4}+12 C_{0}^{1 / 2} C_{f}^{2}$. Therefore, combining (7.4) and (7.6) 
(7.8), we have

$$
I(t) \leq C \varepsilon^{2}\left(t^{1+3 / 4}+t^{1+1 / 2}\right), \quad \text { for } t>0,
$$

where $C=7 C^{\prime 3 / 4} C_{1}^{1 / 4}+18 C^{\prime 1 / 2} C_{f}^{2}$. The desired estimate (7.3) follows from (7.9).

\section{§ 8. Proof of main theorem}

We are ready to give the Proof of Theorem 2.2. By Proposition 7.2, from every subsequence $\left\{\varepsilon^{\prime} \rightarrow 0\right\}$ of $\{\varepsilon\}$, we can find further subsequence $\left\{\varepsilon^{\prime \prime} \rightarrow 0\right\}$ such that $P_{\varepsilon^{\prime \prime}}$ converges weakly to a certain probability distribution $P$ on $\mathscr{C}$. Define $\sigma$-fields $\mathscr{M}_{t}$ and $\mathscr{M}$ on $\mathscr{C}$ as follows:

$$
\begin{aligned}
\mathscr{M}_{t} & =\sigma\left((V(s), f): 0 \leq s \leq t, f \in \mathscr{E}_{r}, V \in \mathscr{C}\right), \\
\mathscr{M} & =\sigma\left(\bigcup_{t \geq 0} \mathscr{M}_{t}\right) .
\end{aligned}
$$

Here $V(s) \in \mathscr{E}_{r}^{\prime}$ is the evaluation of $V$ at time $s$. For each $f \in \mathscr{E}_{r}$ and $t \geq 0$, consider a function $M_{\varepsilon}(t, f)$ on $\mathscr{C}$ :

$$
\begin{aligned}
M_{\varepsilon}(t, f)(V)= & (V(t), f)-(V(0), f) \\
& -\int_{0}^{t}\left(\varepsilon^{-1 / 2}\left(U^{\prime}\left(\varepsilon^{1 / 2} V(s, x)+\rho^{\prime}(\lambda)\right), \Delta_{\varepsilon} f(x)\right) d x, V \in \mathscr{C} .\right.
\end{aligned}
$$

Then, from (3.1), we have

$$
\begin{aligned}
M_{\varepsilon}(t, f)\left(V_{\varepsilon}\right) & =\sqrt{2} \int \nabla_{\varepsilon}^{*} f(x) d w_{\varepsilon}(t, x) \\
& =\sqrt{2 \varepsilon} \sum_{k=-\infty}^{\infty} \int_{\varepsilon k}^{\varepsilon(k+1)} \nabla_{\varepsilon}^{*} f(x) d x \beta\left(t / \varepsilon^{2}, k\right) .
\end{aligned}
$$

This means $M_{\varepsilon}(t, f)$ is the Brownian motion with variance

$$
\frac{2}{\varepsilon} \sum_{k=-\infty}^{\infty}\left(\int_{\varepsilon k}^{s(k+1)} \nabla_{\varepsilon}^{*} f(x) d x\right)^{2}
$$

defined on the probability space $\left(\mathscr{C}, \mathscr{M}, P_{\varepsilon}\right)$. Consequently,

$$
M_{\varepsilon}(t, f) \text { and } M_{\varepsilon}(t, f)^{2}-\frac{2}{\varepsilon} \sum_{k=-\infty}^{\infty}\left(\int_{\varepsilon k}^{\varepsilon(k+1)} \nabla_{\varepsilon}^{*} f(x) d x\right)^{2} t
$$

are $\left(P_{\varepsilon}, \mathscr{M}_{t}\right)$-martingales. Therefore,

$$
\boldsymbol{E}^{p_{\varepsilon}}\left[\left(M_{\varepsilon}(t, f)-M_{\varepsilon}(s, f)\right) \Phi(V)\right]=0,
$$

for $0<s<t$ and each $\mathscr{M}_{s}$-measurable bounded and continuous function $\Phi: \mathscr{C} \rightarrow \boldsymbol{R}$. Let us denote 


$$
\begin{gathered}
I_{1}(\varepsilon)=\boldsymbol{E}^{p_{s}}\left[\left\{(V(t), f)-(V(s), f)-\int_{s}^{t} \rho^{\prime \prime}(\lambda)^{-1 / 2}(V(u), \Delta f) d u\right\} \Phi(V)\right], \\
I_{2}(\varepsilon)=\boldsymbol{E}^{p_{s}}\left[\left\{\int_{s}^{t} \rho^{\prime \prime}(\lambda)^{-1 / 2}\left(V(u), \Delta f-\Delta_{\varepsilon} f\right) d u\right\} \Phi(V)\right] \\
I_{3}(\varepsilon)=\boldsymbol{E}^{p_{\varepsilon}}\left[\left\{\int _ { s } ^ { t } \left(\varepsilon^{-1 / 2} U^{\prime}\left(\varepsilon^{1 / 2} V(u, x)+\rho^{\prime}(\lambda)\right)\right.\right.\right. \\
\left.\left.\left.-\rho^{\prime \prime}(\lambda)^{-1} V(u, x), \Delta_{\varepsilon} f(x)\right) d u\right\} \Phi(V)\right] .
\end{gathered}
$$

Then

$$
\boldsymbol{E}^{p_{\varepsilon^{\prime \prime}}}\left[\left(M_{\varepsilon^{\prime \prime}}(t, f)-M_{\varepsilon^{\prime \prime}}(s, f)\right) \Phi(V)\right]=I_{1}\left(\varepsilon^{\prime \prime}\right)+I_{2}\left(\varepsilon^{\prime \prime}\right)+I_{3}\left(\varepsilon^{\prime \prime}\right) .
$$

Now take the limit $\varepsilon^{\prime \prime} \rightarrow 0$ in (8.4). For $I_{1}$, since $p_{\varepsilon^{\prime \prime}} \rightarrow p$ weakly on $\mathscr{C}$, we have

$$
I_{1}\left(\varepsilon^{\prime \prime}\right) \rightarrow \boldsymbol{E}^{p}[(M(t, f)-M(s, f)) \Phi(V)]
$$

where $M(t, f) \equiv M(t, f)(V)=(V(t), f)-(V(0), f)-\rho^{\prime \prime}(\lambda)^{-1} \int_{0}^{t}(V(u), \Delta f) d u$. For $I_{2}$, it is easy to check that

$$
\left|I_{2}(\varepsilon)\right| \leq \rho^{\prime \prime}(\lambda)^{-1}\|\Phi\|_{\infty}(t-s) \int\left|x-\rho^{\prime}(\lambda)\right| q_{\lambda}(x) d x \varepsilon^{-1 / 2} \int\left|\Delta f(x)-\Delta_{\varepsilon} f(x)\right| d x .
$$

However, since $f \in \mathscr{E}_{r}, \varepsilon^{-1 / 2} \int\left|\Delta f(x)-\Delta_{\varepsilon} f(x)\right| d x \rightarrow 0$ as $\varepsilon \rightarrow 0$. Thus

$$
I_{2}(\varepsilon) \rightarrow 0 \text {. }
$$

For $I_{3}$, we have by Proposition 3.1

$$
I_{3}(\varepsilon) \rightarrow 0 \text {. }
$$

Combining (8.4) (8.7) with (8.3), we have

$$
\boldsymbol{E}^{p}[(M(t, f)-M(s, f)) \Phi(V)]=0 .
$$

Hence, $M(t, f)$ is a $\left(P, \mathscr{M}_{t}\right)$-martingale. And by the similar method, we can show that $M(t, f)^{2}-2\|\nabla f\|^{2} t$ is a $\left(P, \mathscr{M}_{t}\right)$-martingale, too. These imply that $M(t, f)$ is Brownian motion with variance $2\|\nabla f\|^{2}$ for each $f \in \mathscr{E}_{r}$.

For any $\left(a_{1}, \cdots, a_{m}\right) \in \boldsymbol{R}^{m}, m \in N, t_{0}=0 \leq t_{1} \leq \cdots \leq t_{m}, f_{1}, \cdots, f_{m} \in \mathscr{E}_{r}$, a simple computation gives that

$$
\sum_{k=1}^{m} a_{k} M\left(t_{k}, f_{k}\right)=\sum_{k=1}^{m}\left\{M\left(t_{k}, a_{k} f_{k}+\cdots+a_{m} f_{m}\right)-M\left(t_{k-1}, a_{k} f_{k}+\cdots+a_{m} f_{m}\right)\right\} .
$$

Noting that the r.h.s. is a sum of independent Gaussian random variables, we know that the linear combination $\sum_{k=1}^{m} a_{k} M\left(t_{k}, f_{k}\right)$ has a Gaussian 
distribution with respect to $P$. Therefore, $\{M(t, f)\}_{t \geq 0, f \in \delta r}$ is a Gaussian system and one can check that its mean is zero and covariance is

$$
\boldsymbol{E}^{p}[M(t, f) M(s, g)]=2(\nabla f, \nabla g) t \wedge s, \quad \text { for } t, s \geq 0 \text { and } f, g \in \mathscr{E}_{r} .
$$

On the other hand, it is easy to see (cf. [11]) that $V(0)$ is an $\mathscr{E}_{r}^{\prime}$ valued Gaussian random variable under $P$ with mean zero and covariance

$$
\boldsymbol{E}^{p}[(V(0), f)(V(0), g)]=\rho^{\prime \prime}(\lambda)(f, g), \quad f, g \in \mathscr{E}_{r} .
$$

For $V \in \mathscr{C}$, define $\tilde{V} \in \mathscr{C}$ such that

$$
\begin{aligned}
(\tilde{V}(t), f)= & \left(V(0), e^{t \theta \Delta} f\right)+M(t, f)(V) \\
& \left.+\theta \int_{0}^{t} M(s, e)^{(t-s) \theta \Delta} \Delta f\right)(V) d s, \quad f \in \mathscr{E}_{r}
\end{aligned}
$$

where $\theta=\rho^{\prime \prime}(\lambda)^{-1}$. Then, from (8.8) and (8.9), $\left\{\left(\tilde{V}_{t}, f\right)\right\}_{t \geq 0, f \in e_{r}}$ is a Gaussian system with mean zero and covariance

$$
\boldsymbol{E}^{p}[(\tilde{V}(t), f)(\tilde{V}(s), g)]=\rho^{\prime \prime}(\lambda)\left(f, e^{|t-s| \rho^{\prime \prime}(\lambda)-1} g\right) .
$$

However, $\tilde{V}=V, P$-a.s from Lemma 8.1 below and therefore $P$ is independent of the selection of $\left\{\varepsilon^{\prime}\right\}$. This means that $P_{\varepsilon}$ itself converges to $P$ weakly. Since the distribution of the solution of (2.11) coincides with $P$, we have shown the conclusion of Theorem 2.2.

Finally, we prove the lemma used above.

LEMMA 8.1. $\quad P(\tilde{V}=V)=1$.

Proof. First we check that $\tilde{V}$ satisfies

$$
(\tilde{V}(t), f)=(V(0), f)+\rho^{\prime \prime}(\lambda)^{-1} \int_{0}^{t}(\tilde{V}(u), \Delta f) d u+M(t, f)(V) .
$$

This equality also holds for $V(t)$ instead of $\tilde{V}(t)$. To conclude the proof, it is sufficient to show that

$$
\boldsymbol{E}^{p}[\mid(\tilde{V}(t), f)-(V(t), f)]=0, \quad \text { for all } t>0 \text { and } f \in A,
$$

where $A$ is a dense subset of $\mathscr{E}_{r}$. Set $\hat{V}(t)=\tilde{V}(t)-V(t)$. Then $\hat{V}$ satisfies the following equation with probability one:

$$
(\hat{V}(t), f)=\rho^{\prime \prime}(\lambda)^{-1} \int_{0}^{t} d s(\hat{V}(s), \Delta f), \quad \text { for } t>0, f \in \mathscr{E}_{r} .
$$

However, $V(t)$ is stationary under $P$ and also $\tilde{V}(t)$; see (8.10). We therefore have from (8.9) 


$$
\begin{aligned}
\boldsymbol{E}^{p}[|(\hat{V}(t), f)|] & \leq \rho^{\prime \prime}(\lambda)^{-1} \int_{0}^{t} d s \boldsymbol{E}^{p}[|(\hat{V}(s), \Delta f)|] \\
& \leq \sqrt{2} \rho^{\prime \prime}(\lambda)^{-n} \int_{0}^{t} d t_{1} \int_{0}^{t_{1}} d t_{2} \cdots \int_{0}^{t_{n-1}} d t_{n} \boldsymbol{E}^{p}\left[\left(V(0), \Delta^{n} f\right)^{2}\right]^{1 / 2} \\
& \leq \sqrt{2} \rho^{\prime \prime}(\lambda)^{-n+1 / 2} t^{n}\left\|\Delta^{n} f\right\|_{\boldsymbol{L}^{2}(\boldsymbol{R})} / n !
\end{aligned}
$$

We take $A$ to be the linear hull of $\left\{h_{m} e^{-r \xi(x)} ; m \in N\right\}$, where $h_{m}(x)=$ $\left(2^{m} m ! \sqrt{\pi}\right)^{-1 / 2} e^{-x / 2} H_{m}(x)$, and $H_{m}(x), m=0,1,2, \cdots$ are the Hermite polynomials. One can check that $C^{n}\left\|\Delta^{n} h_{m}\right\|^{2} / n !=o(1)$ as $n \rightarrow \infty$ for all $m \in N$ with some constant $C$. This implies (8.11).

Acknowledgement. The author wishes to thank Professors T. Hida and T. Funaki for valuable suggestions and kind encouragements.

\section{REFERENCES}

[1] Fritz, J., On the hydrodynamic limit of a scalar Ginzburg-Landau lattice model, The resolvent approach, in: Hydrodynamic Behavior and Interacting particle system, IMA volumes in Math. Appl., 9, Papanicolaou (ed.), 75-97.

[2] —-, On the hydrodynamic limit of a Ginzburg-Landau lattice model, The a priori bounds, J. Statis. Phys., 47 (1987), 551-572.

[ 3 ] Guo, M. Z., Papanicolaou, G. C. and Varadhan, S. R. S., Nonlinear diffusion limit for a system with nearest neighbor interactions, Commun. Math. Phys., 118 (1988), $31-59$.

[4] Mitoma, I., Tightness of probabilities on $C\left([0,1] ; \mathscr{S}^{\prime}\right)$ and $D\left([0,1] ; \mathscr{S}^{\prime}\right)$, The Annals of Probability, 11, No. 4, (1983) 989-999.

[ 5 ] Petrov, V. V., Sums of Independent Random Variables, Springer-Verlag, Berlin, Heidelberg, New York, 1975.

[ 6 ] Reed, M. and Simon, B., Methods of modern mathematical Physics, Vol. II. New York: Academic Press 1970.

[ 7 ] Rost, H., Hydrodynamik gekoppelter Diffusionen: Fluktuationen im Gleichgewicht, in: Lecture Notes in Mathematics, Vol. 1031, Berlin, Heidelberg, New York: Springer 1983.

[ 8 ] - On the behavior of the hydrodynamical limit for stochastic particle systems, Lect. Note Math., 1215, 129-164.

[9] Shiga, T. and Shimizu, A., Infinite dimensional stochastic differential equations and their applications, J. Math. Kyoto Univ. (JMKYAZ), 20-3 (1980), 395-416.

[10] Spohn, H., Equilibrium fluctuations for interacting Brownian particles, Commun. Math. Phys., 103 (1986), 1-33.

[11] Zhu, M., The central limit theorem for a scalar Ginzburg-Landau equation, stationary case (in Japanese), Master thesis, Nagoya Univ., 1987.

Department of Mathematics

School of Science

Nagoya University

Chikusa-ku, Nagoya, 464-01, Japan 